\title{
Genome-wide identification and functional analysis of circRNAs in Trichophyton rubrum conidial and mycelial stages
}

\author{
Xingwei $\mathrm{CaO}^{\dagger}$, Xingye $\mathrm{Xu}^{\dagger}$, Jie Dong, Ying Xue, Lilian Sun, Yafang Zhu, Tao Liu* and Qi Jin*
}

\begin{abstract}
Background: Circular RNAs (circRNAs) are a group of noncoding RNAs that participate in gene expression regulation in various pathways. The essential roles of circRNAs have been revealed in many species. However, knowledge of circRNAs in fungi is still not comprehensive.

Results: Trichophyton rubrum (T. rubrum) is considered a model organism of human pathogenic filamentous fungi and dermatophytes. In this study, we performed a genome-wide investigation of circRNAs in T. rubrum based on high-throughput sequencing and ultimately identified 4254 circRNAs. Most of these circRNAs were specific to the conidial or mycelial stage, revealing a developmental stage-specific expression pattern. In addition, 940 circRNAs were significantly differentially expressed between the conidial and mycelial stages. PCR experiments conducted on seven randomly selected differentially expressed (DE-) circRNAs confirmed the circularized structures and relative expression levels of these circRNAs. Based on their genome locations, most circRNAs originated from intergenic regions, unlike those in plants and animals. Furthermore, we constructed circRNA-miRNA-mRNA regulatory networks that included 661 DE-circRNAs targeting 140 miRNAs and further regulating 2753 mRNAs. The relative expression levels of two randomly selected circRNA-miRNA-mRNA axes were investigated by qRT-PCR, and the competing endogenous RNA (ceRNA) network theory was validated. Functional enrichment analysis of the target genes suggested that they were significantly involved in posttranscriptional processes and protein synthesis as well as some small-molecule metabolism processes. CircRNAs are relatively more conserved in closely related dermatophytes but rarely conserved in distantly related species. Tru_circ07138_001 is a highly conserved circRNA that was conserved in all ten dermatophytes analyzed in our study and three distantly related species. Its host gene TERG_07138 was also highly conserved in two of these distantly related species Gallus gallus and Caenorhabditis elegans. The specific role of this circRNA deserves further exploration.
\end{abstract}

Conclusions: Our study is the first to provide a global profile of circRNAs in T. rubrum as well as dermatophytes. These results could serve as valuable resources for research on circRNA regulatory mechanisms in fungi and reveal new insights for further investigation of the physical characteristics of these significant human fungal pathogens.

Keywords: Trichophyton rubrum (T. rubrum), High-throughput sequencing, Circular RNA (circRNA), microRNA (miRNA), Target gene

*Correspondence: liutao@ipbcams.ac.cn; zdsys@vip.sina.com ${ }^{+}$Xingwei Cao and Xingye Xu contributed equally to this work. NHC Key Laboratory of Systems Biology of Pathogens, Institute of Pathogen Biology, Chinese Academy of Medical Sciences \& Peking Union Medical College, Beijing, P. R. China

\section{Background}

Circular RNAs (circRNAs) are a group of endogenous noncoding RNAs (ncRNAs) that are each composed of a single-stranded covalently bonded ring structure [1, 2]. In 1976, Sanger et al. first discovered the existence of 
circRNAs in plant viroids [3]. In the following decades, circRNAs were also detected in other viruses and human cells $[4,5]$. Due to the limitations of sequencing and analysis methods, circRNAs were originally mistaken as rare viroid-characteristic or useless RNAs produced by abnormal RNA splicing [6]. Recently, studies on circRNAs have achieved great progress given the rapid advances in highthroughput sequencing technology; the development of a growing number of circRNA identification tools, such as CircInteractome [7], circRNA_finder [8], CircRNA Identifier (CIRI) [9], find_circ [10] and CIRCexplorer [11]; and the establishment of circRNA annotation databases, including CircNet [12], Circ2Traits [13] and deepBase v2.0 [14].

CircRNAs are generated by back-splicing of precursor mRNA (pre-mRNA) [1]. In contrast to canonical splicing, which joins the upstream $5^{\prime}$ splice (donor) site with a downstream $3^{\prime}$ splice (acceptor) site to form a linear RNA transcript, back-splicing connects a downstream $5^{\prime}$ splice site with an upstream $3^{\prime}$ splice site, forming a covalently closed circRNA transcript [15]. Due to their ring structures, circRNAs are more stable and less prone to degradation than linear transcripts [16]. CircRNAs are essential transcription regulators that are considered microRNA (miRNA) sponges that competitively regulate target gene expression [17]. In addition, circRNA biogenesis may compete with host pre-mRNA linear splicing, and some circRNAs with internal ribosomal entry sites (IRESs) and $\mathrm{N}^{6}$-methyladenosine $\left(\mathrm{m}^{6} \mathrm{~A}\right)$ have translational potential $[1,18]$. The essential roles of circRNAs have been illustrated in many species, including humans [19], mice [20], zebrafish [21], Caenorhabditis elegans [22], Drosophila [8], Saccharomyces cerevisiae [23], Arabidopsis thaliana [24], rice [25] and soybean [26].

CircRNAs in fungi have been researched in only a few studies. In 2018, Guo et al. identified 551 circRNAs in Ascosphaera apis, most of which ranged in length from 200 to $600 \mathrm{nt}$, unlike circRNAs in animals and plants. These circRNAs play vital roles in metabolism, environmental stress and gene expression [27]. In 2018, Yuan et al. detected 2721 and 5840 circRNAs in conidial and mycelial samples, respectively, of the plant pathogenic fungus Magnaporthe oryzae, which infects rice. The host genes of circRNAs in conidia were involved mainly in the synthesis of storage products for rice infection, while those of circRNAs in mycelia were involved primarily in basic metabolism for the promotion of normal fungal growth [28]. In addition, Guo et al. identified 204 circRNAs in spore samples of the microsporidium species Nosema ceranae, and circRNA-miRNA-mRNA regulatory networks showed that the target genes were engaged in various metabolic pathways, indicating that these circRNAs might play regulatory roles in $N$. ceranae spores
[29]. However, knowledge about circRNAs in fungi remains from sufficient.

Trichophyton rubrum (T. rubrum) is the most common pathogen responsible for fungal infections worldwide and has been studied as a model organism of dermatophytes and human pathogen filamentous fungi [30-32]. In addition to dermatomycosis, deep infections caused by $T$. rubrum have been occasionally reported and threaten the lives of immune-deficient patients [33, 34]. The incidence of infections caused by T. rubrum continues to rise, and these infections are receiving increasing attention [35]. Resistance to antifungal reagents and frequent relapses are also great concerns in therapy [36]. T. rubrum has two major developmental stages in its life cycle: the conidial stage, in which the fungus adheres to the stratum corneum of skin, and the mycelial stage, in which the fungus penetrates and destroys the superficial layers [37]. The biological characteristics and pathogenic mechanisms of T. rubrum have been extensively studied at the molecular level, which has improved our understanding of this fungus [38-43]. The characteristics and roles of circRNAs in T. rubrum remain unknown.

In the current study, we performed global profiling of circRNAs in T. rubrum based on high-throughput RNA sequencing (RNA-seq). A total of 4254 circRNAs were identified in both conidial and mycelial stages, and 940 circRNAs were considered to be significantly differentially expressed between the two stages. With further bioinformatics analysis, the genomic location, alternative splicing (AS) pattern, conservation and regulatory roles of circRNAs were also investigated. These results provide new clues for research on circRNA regulation mechanisms in fungi and could serve as a foundation for further studies on these medically important pathogenic fungi.

\section{Results \\ Overview of high-throughput sequencing data for $T$. rubrum}

To identify distinct patterns of circRNAs in the two major growth stages of T. rubrum, we constructed cDNA libraries of conidia and mycelia with three biological replicates and sequenced them with an Illumina HiSeq platform. After the adaptors were trimmed and the low-quality reads or reads with unknown $(\mathrm{N})$ bases were filtered out, averages of 125.1749 million and 124.6443 million clean reads were obtained for the conidial and mycelial stages, respectively (Table 1 ). All clean reads were mapped to the T. rubrum reference genome, and the mean mapping rate for the six samples was $97.6 \%$. In addition, the average values of Pearson's correlation coefficient were 0.9385 and 0.8239 for circRNA expression levels in conidia and mycelia, respectively (Additional file 1: Fig. S1), highlighting good consistency among the three replicates in 
Table 1 Summary of RNA-seq data for T. rubrum conidial and mycelial samples

\begin{tabular}{llllll}
\hline Sample & Clean reads & GC (\%) & Q30 (\%) & Mapped reads & Mapping rate (\%) \\
\hline Conidia_1 & $122.5520 \mathrm{M}$ & 46.58 & 90.92 & $121.0295 \mathrm{M}$ & $98.76 \%$ \\
Conidia_2 & $126.8353 \mathrm{M}$ & 46.93 & 90.46 & $123.7974 \mathrm{M}$ & $97.61 \%$ \\
Conidia_3 & $126.1375 \mathrm{M}$ & 46.21 & 91.31 & $120.9987 \mathrm{M}$ & $95.93 \%$ \\
Mycelia_1 & $124.3186 \mathrm{M}$ & 45.98 & 91.34 & $121.7001 \mathrm{M}$ & $97.90 \%$ \\
Mycelia_2 & $127.2742 \mathrm{M}$ & 44.71 & 89.99 & $123.7542 \mathrm{M}$ & $97.24 \%$ \\
Mycelia_3 & $122.3400 \mathrm{M}$ & 45.51 & 90.68 & $120.1093 \mathrm{M}$ & $98.18 \%$ \\
\hline
\end{tabular}

each stage. Together, these results suggest the reliability and repeatability of our sequenced data.

\section{Identification and characterization of circRNAs}

A total of 4254 circRNAs (Additional file 2: Table S1) were identified in T. rubrum, including 3980 in conidia and 778 in mycelia. Most of the identified circRNAs were growth stage specific; 3476 circRNAs were specific to conidia, and 274 circRNAs were specific to mycelia.

To obtain an overview of circRNA distribution within the T. rubrum genome, we investigated the locations of circRNAs on each genomic contig (Table 2). The numbers of circRNAs on distinct genomic contigs were significantly different. This difference may have been due to the length differences of distinct contigs.
We also created a Circos plot to directly visualize the distribution of circRNAs in the whole genome of $T$. rubrum. As shown in Fig. 1, circRNAs were evenly distributed on all contigs, suggesting that circRNAs have no preference for genomic regions.

In addition, the identified circRNAs could be classified into three groups, including exonic, intronic and intergenic circRNAs, according to their genomic locations. Intergenic circRNAs were the most abundant (60.5\%), followed by exonic circRNAs (35.2\%) and intronic circRNAs (4.3\%). When the conidial and mycelial stages were compared, these three kinds of circRNAs showed similar proportions in the distribution (Fig. 2a).

The lengths of circRNAs varied greatly. The longest circRNA was $196,395 \mathrm{nt}$, and the shortest was $20 \mathrm{nt}$. As

Table 2 Distribution of circRNAs and back-spliced reads in the whole genome of T. rubrum

\begin{tabular}{lllll}
\hline Genomic region & CircRNA number & CircRNA percentage & No. of back-spliced reads & Read percentage \\
\hline NW_003456399.1 & 1 & $0.02 \%$ & 4 & $0.01 \%$ \\
NW_003456411.1 & 7 & $0.16 \%$ & 19 & $0.07 \%$ \\
NW_003456412.1 & 29 & $0.68 \%$ & 101 & $0.38 \%$ \\
NW_003456413.1 & 10 & $0.24 \%$ & 128 & $0.48 \%$ \\
NW_003456414.1 & 32 & $0.75 \%$ & 149 & $0.55 \%$ \\
NW_003456415.1 & 36 & $0.85 \%$ & 187 & $0.69 \%$ \\
NW_003456416.1 & 37 & $0.87 \%$ & 144 & $0.53 \%$ \\
NW_003456417.1 & 93 & $2.19 \%$ & 566 & $2.10 \%$ \\
NW_003456418.1 & 192 & $4.51 \%$ & 1008 & $3.73 \%$ \\
NW_003456419.1 & 152 & $3.57 \%$ & 854 & $3.16 \%$ \\
NW_003456420.1 & 232 & $5.45 \%$ & 1450 & $5.37 \%$ \\
NW_003456421.1 & 243 & $5.71 \%$ & 1155 & $4.28 \%$ \\
NW_003456422.1 & 332 & $7.80 \%$ & 2167 & $8.03 \%$ \\
NW_003456423.1 & 288 & $6.77 \%$ & 1585 & $5.87 \%$ \\
NW_003456424.1 & 353 & $8.30 \%$ & 2233 & $8.27 \%$ \\
NW_003456425.1 & 432 & $10.16 \%$ & 3943 & $14.60 \%$ \\
NW_003456426.1 & 457 & $10.74 \%$ & 2523 & $9.35 \%$ \\
NW_003456427.1 & 457 & $10.74 \%$ & 2347 & $8.69 \%$ \\
NW_003456428.1 & 853 & $20.06 \%$ & 6341 & $23.49 \%$ \\
NW_003456429.1 & 18 & $0.43 \%$ & 95 & $0.35 \%$ \\
Total & 4254 & $100.00 \%$ & 26,999 & $100.00 \%$ \\
\hline
\end{tabular}




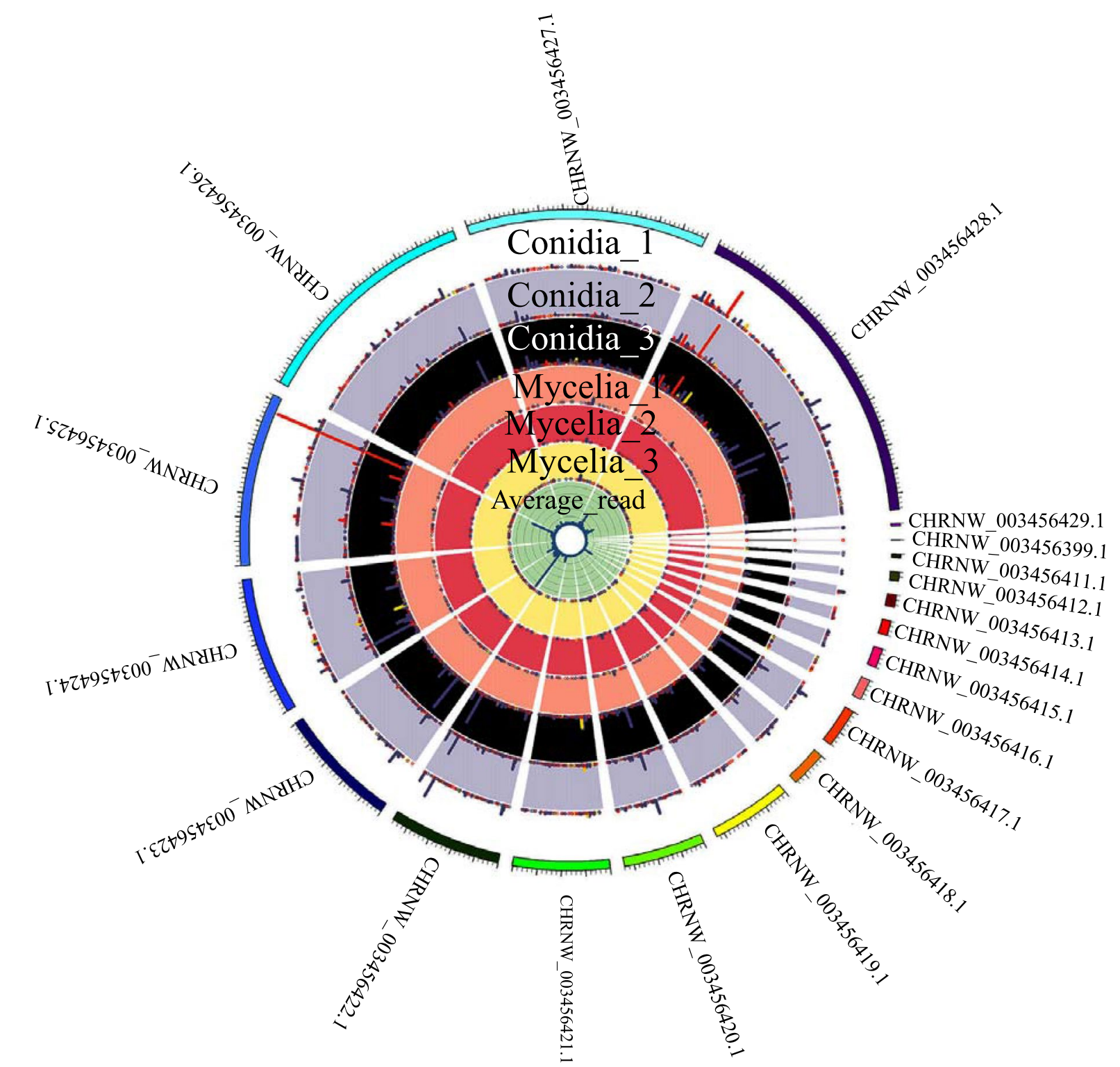

Fig. 1 The Circos plot shows the distribution and abundance of circRNAs in the whole genome. The red bar represents exonic circRNAs, the yellow bar represents intronic circRNAs and the blue bar represents intergenic circRNAs. The height of each bar indicates the read number for each circRNA. The outermost layer represents genomic contigs. The six layers in the middle represent the three replicates in each stage, including conidia replicate 1, conidia replicate 2 , conidia replicate 3 , mycelia replicate 1, mycelia replicate 2 and mycelia replicate 3 . The innermost layer shows the number of average junction reads per circRNA of these six samples

shown in Fig. 2b, $61.36 \%$ of circRNAs were shorter than $500 \mathrm{nt}, 26.3 \%$ were within $500-1000 \mathrm{nt}$, and $12.34 \%$ were longer than $1000 \mathrm{nt}$. The average length of all the identified circRNAs was $639 \mathrm{nt}$.

\section{Identification of differentially expressed (DE-) circRNAs}

CircRNAs identified in the conidial and mycelial stages were compared to identify DE-circRNAs. Based on the criteria of $\mid \log _{2}$ (fold change) $\mid \geq 1$ and probability $\geq 0.8[44,45], 940$ circRNAs were considered to be significantly differentially expressed, including 930 up-regulated and 10 down-regulated circRNAs in the conidial vs. mycelial stage (Fig. 3). Detailed information on these DE-circRNAs is described in Additional file 3: Table S2. The most significantly up-regulated circRNAs were Tru_circ00222_002, Tru_circ_sc2.12_00022 and Tru_circ_sc2.5_00054, which had $\mid \log _{2}$ (fold change) $\mid$ 

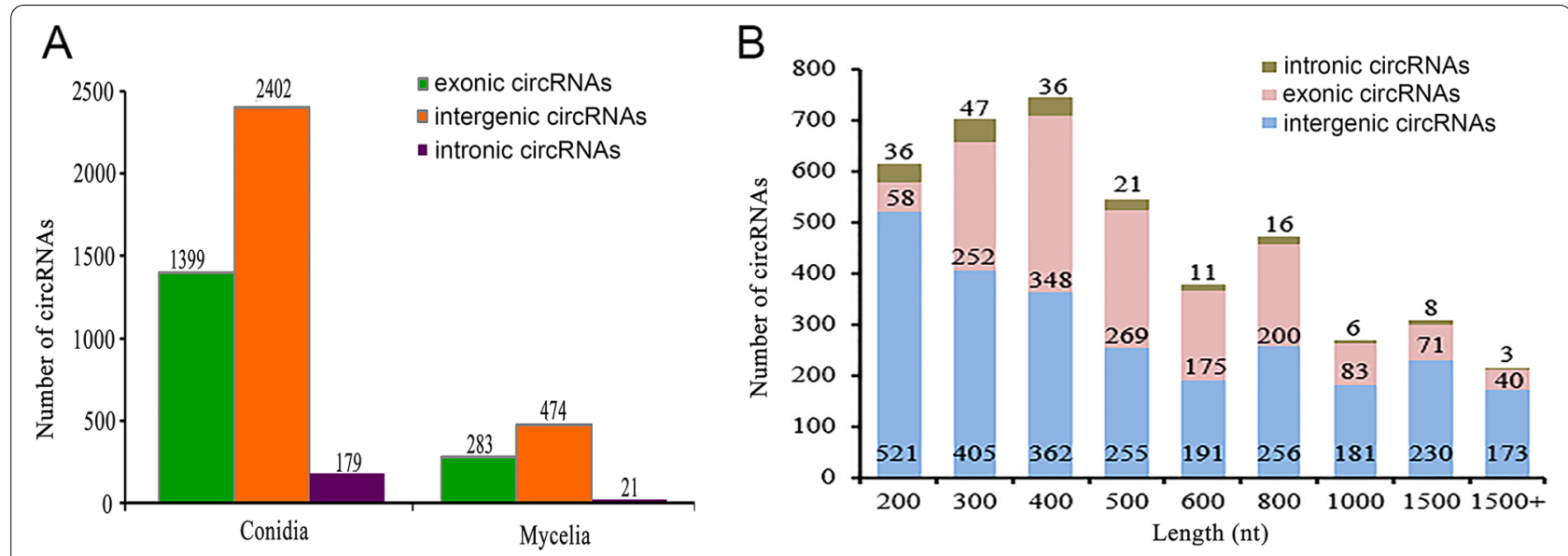

Fig. 2 Type and length of all the identified circRNA. a The number of each type of circRNA in the conidial and mycelial stages. $\mathbf{b}$ Length distribution of intronic, intergenic and exonic circRNAs

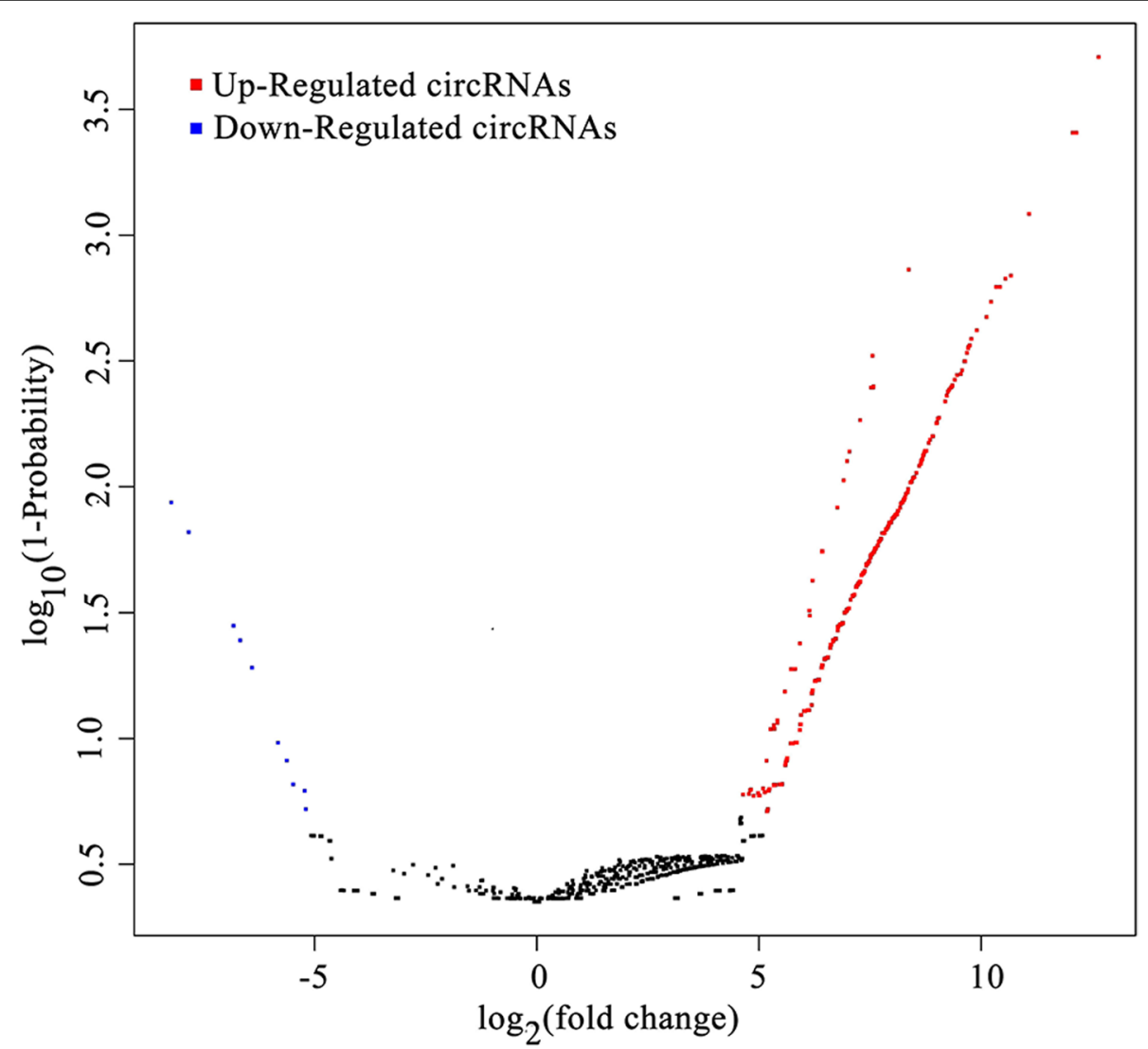

Fig. 3 Volcano plot of circRNA relative expression levels in the conidial vs. mycelial stage. Red dots represent up-regulated circRNAs and blue dots represent down-regulated circRNAs in conidia vs. mycelia. Black dots represent circRNAs that are not significantly differentially expressed between the two stages 


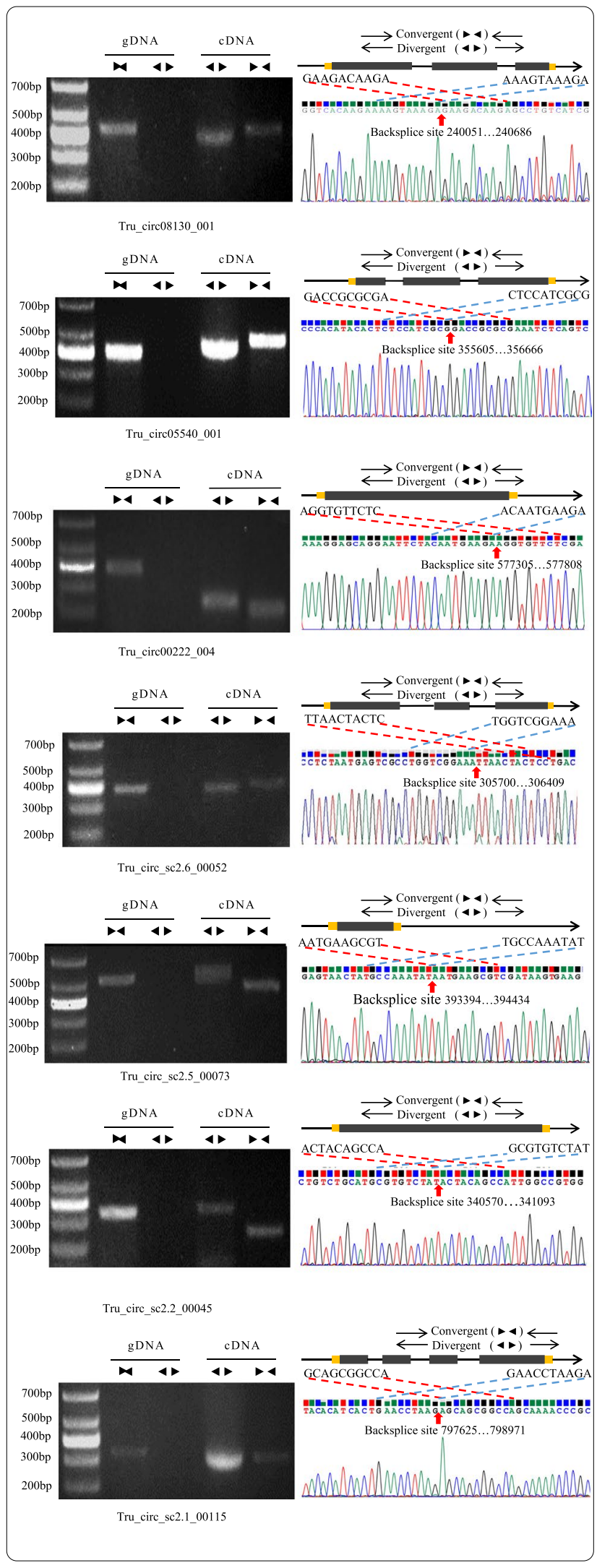

Fig. 4 Validation of the circularized structure of circRNAs. Seven randomly selected DE-circRNAs were confirmed by PCR experiments with divergent and convergent primers. The amplification products were tested by agarose gel electrophoresis. Each cropped gel shows the bands that were amplified with divergent and convergent primers of both circRNA and gDAN control. The back-splicing junctions of circRNA were further confirmed by Sanger sequencing

values of $12.64,12.14$ and 12.12 , respectively, while the most significantly down-regulated circRNAs were Tru_circ05540_001, Tru_circ08130_001, and Tru_circ sc2.6_00055, which had $\mid \log _{2}$ (fold change) $\mid$ values of $8.23,7.83$ and 6.82 , respectively.

Hierarchical cluster analysis additionally showed significantly different expression patterns between up-regulated and down-regulated circRNAs, and suggested good consistency among the three replicates (Additional file 1: Fig. S2).

\section{Validation of the circularized structures and relative expression levels of circRNAs}

To validate that the circRNAs identified in our study were circularized after splicing, we performed PCR experiments on seven randomly selected DE-circRNAs. Seven pairs of convergent and divergent primers were designed for these circRNAs. Theoretically, divergent primers designed to span circRNA back-splicing junctions should specifically amplify circularized RNAs, while convergent primers used as positive controls should amplify linear sequences of both the transcript and genome. As shown in Fig. 4 and Additional file 1: Fig. S3, all reactions using the cDNA template yielded amplification products of the expected sizes with both convergent and divergent primers. Meanwhile, amplification in the control reaction using the gDNA template could only be achieved with the convergent primers; the corresponding bands were not obtained with the divergent primers. To further confirm the fragments amplified by divergent primers spanning the expected back-splicing sites, these seven PCR-amplified products were subjected to Sanger sequencing. All of the sequencing results validated the expected splicing sites.

In addition, we verified the relative expression levels of these seven DE-circRNAs via qRT-PCR using divergent primers. Based on the results of high-throughput sequencing, five circRNAs, Tru_circ00222_004, Tru_circ sc2.6_00052, Tru_circ_sc2.5_00073, Tru_circ_sc2.2_00045 and Tru_circ_sc2.1_00115, were up-regulated in conidia vs. mycelia, while two circRNAs, Tru_circ08130_001 and Tru_ circ05540_001, were down-regulated. As shown in Additional file 1: Fig. S4, the qRT-PCR results for all of these circRNAs exhibited expression patterns consistent with 
those indicated by the high-throughput sequencing data. All of these results demonstrate that the circRNAs identified in our study are highly reliable.

\section{Identification of circRNA host genes and AS events}

Among all the identified circRNAs, 1680 circRNAs were mapped to 1055 host genes. Among the mapped circRNAs, 326 DE-circRNAs corresponded to 249 host genes. To present a comprehensive landscape of circRNA origination, we performed GO and KEGG enrichment analyses for the host genes of all circRNAs (Additional file 4: Table S3, Additional file 5: Table S4, Additional file 1: Fig. S5, S6) and of DE-circRNAs (Additional file 6: Table S5, Additional file 7: Table S6 and Additional file 1: Fig. S7, S8). The results showed that the host genes were involved in various metabolic and biosynthetic processes.

In most instances, one host gene corresponded to one circRNA. In some cases, a host gene corresponded to two or more circRNAs. For instance, in our study, the TERG_08844 gene (Gene ID: 10372936) corresponded to 15 circRNAs. We also found that DE-circRNAs corresponding to identical host genes all showed consistent relative expression differences in the conidial vs. mycelial stage (i.e., they were all up-regulated or down-regulated).

The correspondence of two or more circRNAs to a single host gene may be due to alternative back-splicing events when these circRNAs share identical splicing acceptors or donors [15]. For example, three circRNAs, Tru_circ00222_001, Tru_circ00222_002 and Tru circ00222_004, were determined to be derived from the same host gene, TERG_00222 (Gene ID: 10377559), in the current study. Analysis of the circRNA gene structures showed that Tru_circ00222_001 and Tru circ00222_002 have identical circular ends but that Tru_circ00222_002 includes an extra 504 nt sequence. Tru_circ00222_002 and Tru_circ00222_004 also have identical splicing sites, and the Tru_circ00222_002 sequence is $165 \mathrm{nt}$ longer than the Tru_circ00222_004 sequence (Fig. $5 \mathrm{a}$ ).

AS is a common event that exists in various organisms, greatly expanding the complexity and diversity of the transcriptome [46]. AS events in circRNAs have also been frequently identified in animals and plants, which may facilitate the diversity of circRNAs [47, 48]. To investigate the existence of circRNA AS (circAS) in T. rubrum, we searched all the sequenced samples using the CIRI-AS algorithm to identify circ-AS events. A total of 4 types of circ-AS events, including exon skipping (ES), intron retention (IR), alternative $5^{\prime}$ splice site (A5SS) splicing and alternative $3^{\prime}$ splice site (A3SS) splicing, were identified in 101 circRNAs (Additional file 8: Table S7). Among these, 91 circ-AS events were specific to conidia, and 7 circ-AS events were specific to mycelia. Three circ-AS events were common to both stages. A3SS splicing was the most common circ-AS type, accounting for $59.57 \%$ of all circ-AS events in conidia and $80.0 \%$ of events in mycelia. A5SS splicing, which accounted for $45.74 \%$ of events in conidia and $20.0 \%$ of events in mycelia, was the next most common type. The other two types of circ-AS were rare and were only identified in the conidial stage; IR accounted for $7.45 \%$ of events, and ES accounted for $5.32 \%$ of events (Fig. 5b). These results showed that circ-AS events were much more common in conidia than in mycelia. To examine whether this difference was due to the greater number of circRNAs in conidia than mycelia, we calculated the AS ratio in each stage. The ratio of AS events was $2.4 \%$ in conidia (94 out of 3980 ) and $1.3 \%$ in mycelia (10 out of 778 ), suggesting a greater AS ratio in conidia than in mycelia. What caused this different AS ratio between the two stages deserves further investigation. Whether the differences between the two stages and among the four AS types are due to bias in circRNA identification could be resolved with advances in methodologies.

\section{Construction of a competing endogenous RNA (ceRNA) regulatory network}

Studies have shown that circRNAs competitively bind miRNAs to prevent the binding of miRNAs to mRNAs; thus, these circRNAs are considered ceRNAs that interact with target miRNAs to control target gene expression $[49,50]$. We constructed a ceRNA network based on the DE-circRNAs identified in our study. Among the 940 DEcircRNAs, 661 DE-circRNAs were predicted to target 140 miRNAs (Additional file 9: Table S8), and these miRNAs further regulated 2753 mRNAs (Additional file 10: Table S9). In this network, 207 circRNAs targeted single miRNAs. However, most circRNAs targeted two or more miRNAs, and each miRNA was frequently targeted by more than one circRNA. For example, the circRNA Tru_circ_sc2.10_00071 was predicted to target the most miRNAs (20), followed by Tru_circ_sc2.9_00086 (14 miRNAs) and Tru_circ_sc2.2_00112 (13 miRNAs). In addition, the miRNA Tru-miR-4459 was targeted by the most circRNAs (52), followed by Tru-miR-854 (46 circRNAs) and Tru-miR-762 (45 circRNAs).

Similarly, a single miRNA could target multiple mRNAs. For example, the miRNA Tru-miR-4459 targeted the most mRNAs (406), followed by Tru-miR-595 (354 mRNAs) and Tru-miR-3620-5p (330 mRNAs).

The up-regulated circRNA Tru_circ_sc2.6_00052 was arbitrarily selected to construct a circRNA-miRNAmRNA network. As shown in Fig. 6, the circRNA Tru_circ_sc2.6_00052 targeted six miRNAs, and these miRNAs further regulated 414 mRNAs in total. 


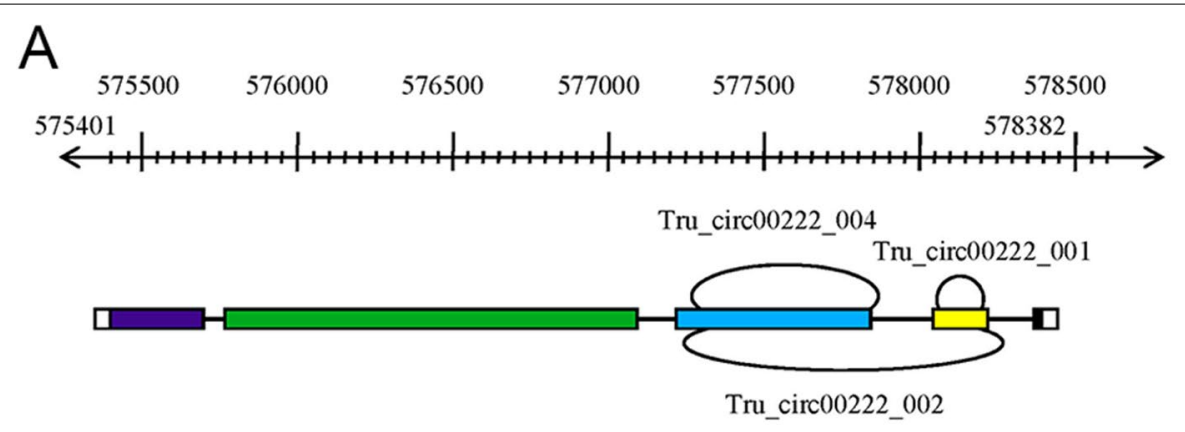

B

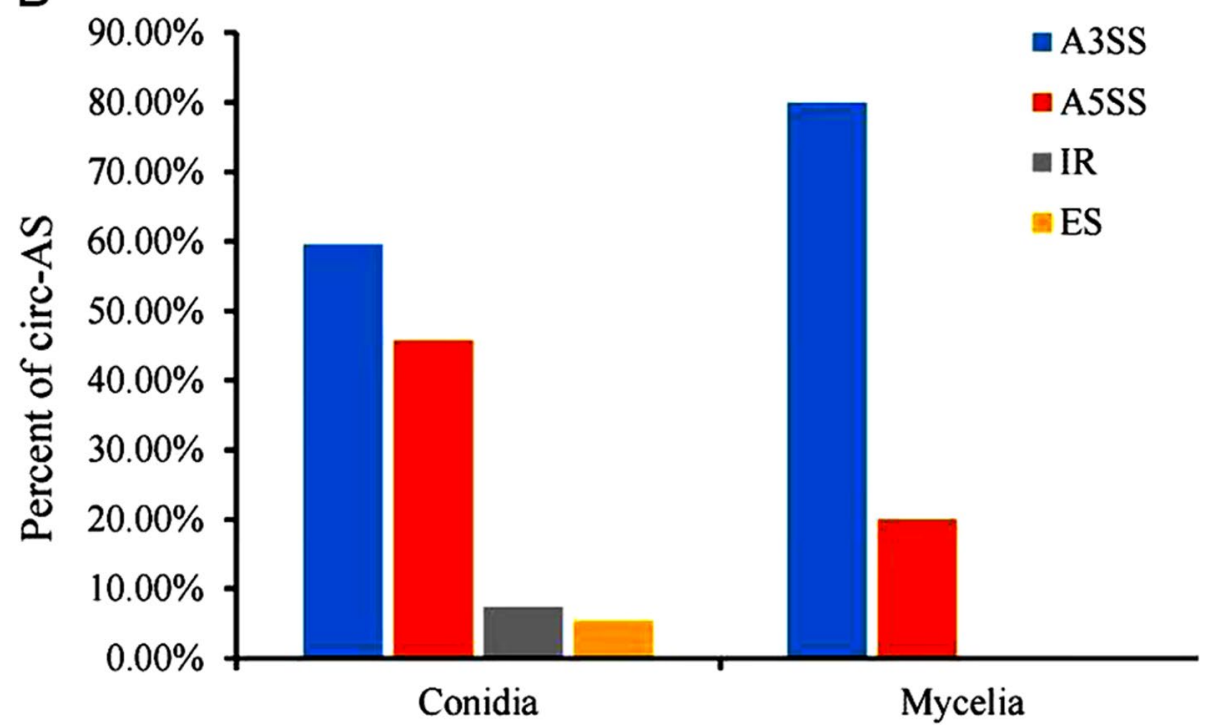

Fig. 5 Alternative splicing events in T. rubrum. a Schematic chart illustrating the formation of three circRNAs, Tru_circ00222_001,Tru_circ00222_002 and Tru_circ00222_004. These three circRNAs are generated by alternative splicing from the same host gene:TERG_00222. b Four types of AS events identified in the conidial and mycelial stages. A3SS: alternative $3^{\prime}$ splice site; A5SS: alternative $5^{\prime}$ splice site; IR: intron retained; ES: exon skipping

These results illustrate the complexity of gene expression regulation. In addition, transcription factors (TFs) have been found to participate in this regulatory process [51]. In our previous study, the miRNA Tru-miR5658 was suggested to bind to three TFs, including the C6 transcription factor, bZIP transcription factor and transcription factor TFIIIB component [52]. In this study, Tru-miR5658 was targeted by 17 up-regulated circRNAs. This result implies that TFs might associate with circRNAs and miRNAs to regulate target gene expression during T. rubrum development.

\section{Functional annotation of target genes regulated by DE-circRNAs in the ceRNA network}

To further uncover the potential roles of circRNAs in the ceRNA network, 2753 target genes regulated by DEcircRNAs were subjected to GO and KEGG enrichment. Based on GO annotation of biological processes, the target genes were heavily involved in ribosome biogenesis, ribonucleoprotein complex biogenesis, rRNA processing, rRNA metabolic processes, and RNA metabolic processes, suggesting their roles in posttranscriptional regulation and protein expression-related pathways. In addition, the target genes participated in small-molecule metabolism, such as small-molecule catabolic processes, nucleobase-containing compound metabolic processes, carboxylic acid metabolic processes and monocarboxylic acid metabolic processes. In the molecular function category, the target genes were significantly involved in acetyltransferase activity and $\mathrm{N}$-acyltransferase activity. In the cellular component category, target genes were significantly associated with the nucleolus, host cellular components and host cell parts (Fig. 7a, Additional file 11: Table S10).

KEGG analysis suggested that the target genes were highly involved in protein synthesis-related processes, 


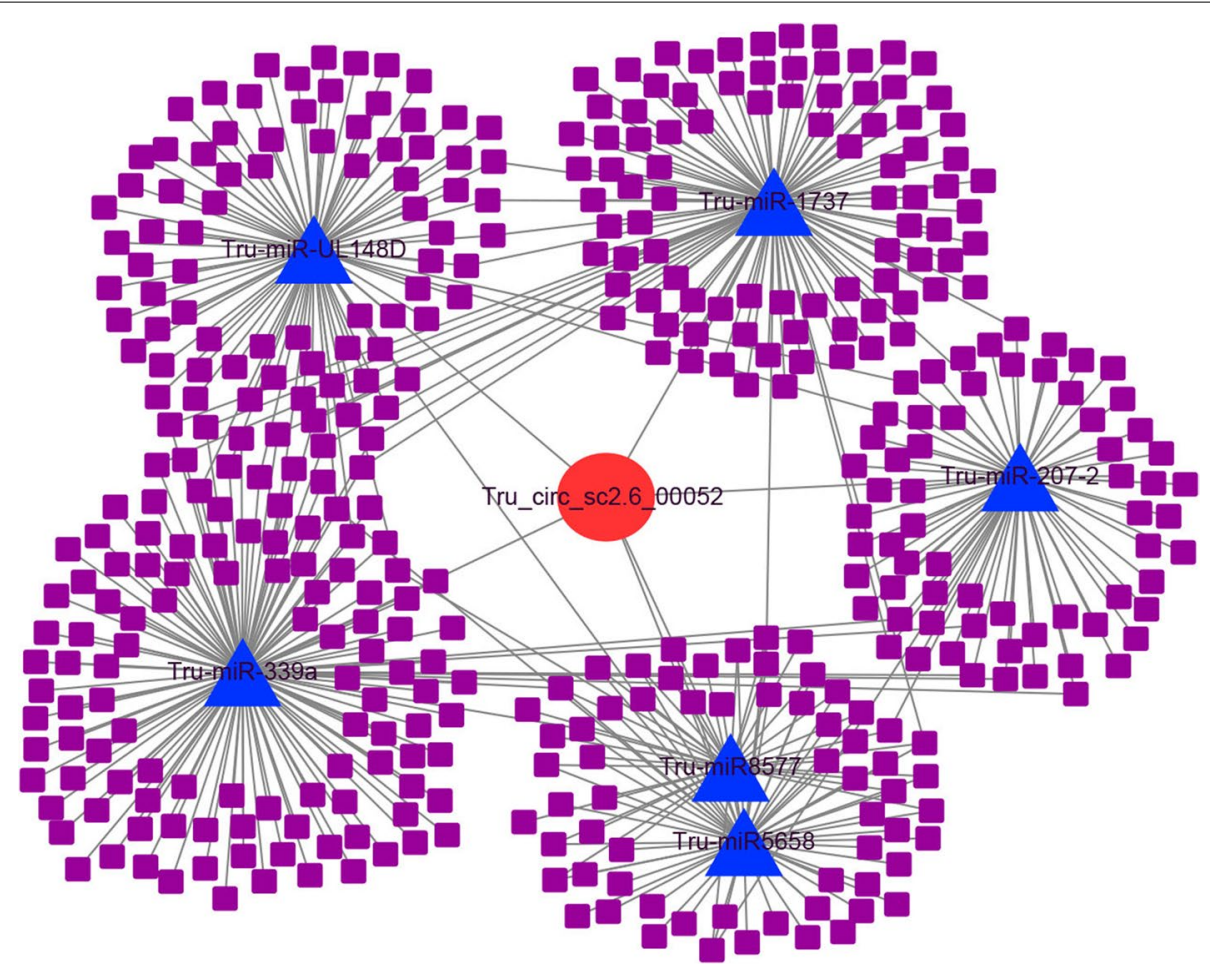

Fig. 6 CeRNA network of circRNA Tru_circ_sc2.6_00052. The red circle represents circRNA; the blue triangle represents miRNA; and the purple square represents mRNA

including RNA degradation and ribosome biogenesis in eukaryotes. Some small-molecule metabolic processes were also enriched, including arginine and proline metabolism, alanine, aspartate and glutamate metabolism, aflatoxin biosynthesis and vitamin B6 metabolism (Fig. 7b, Additional file 12: Table S11). These results are consistent with the results of GO enrichment analysis in the biological process category stated above.

In addition, some target genes are considered to be related to fungal virulence and pathogenicity, including secreted proteases and multidrug efflux transporters (Additional file 13: Table S12). Since dermatophytes are almost always localized in keratinized tissues, proteolytic enzymes and other elements involved in keratin biodegradation are essential virulence factors for these fungi [53-55]. In our study, 31 target genes were considered secreted proteases or peptidases, such as alkaline phosphatase, aspartyl aminopeptidase, extracellular aspartic endopeptidase, subtilisin-like protease and tripeptidyl peptidase SED3. The multidrug efflux pump, which contributes to antifungal resistance, is considered another factor of fungal virulence [56]. Two main families belong to efflux transporters: the major facilitator superfamily (MFS) and ATP-binding cassette (ABC) superfamily [56, 57]. In total, 3 MFS and 6 ABC superfamily transporters were identified in our study.

\section{Investigation of the relative expression levels of two} circRNA-miRNA-mRNA axes

Two circRNA-miRNA-mRNA axes in the ceRNA network were randomly selected to investigate their relative expression levels. For one axis, the qRT-PCR results showed that the miRNA Tru-miR2673a was downregulated, whereas the circRNA Tru_circ_sc2.7_00077 and the target gene TERG_02081 (transcript ID: XM_003237312.1, encoding an RNA-binding protein) were both significantly up-regulated in conidia vs. mycelia (Fig. 8a). For the other axis, the circRNA Tru circ_sc2.6_00029 was predicted to target the miRNA Tru-miR-3113-3p, and Tru-miR-3113-3p was suggested to target three target genes, TERG_08278 (transcript ID: XM_003231143.1, encoding a serine/threonine protein kinase), TERG_02753 (transcript ID: XM_003235649.1, encoding an E3 ubiquitin-protein ligase) and TERG_01198 (transcript ID: XM_003239168.1, encoding the pre-mRNA splicing factor rse1). Based on the qRTPCR results, as shown in Fig. 8b, the circRNA Tru_circ sc2.6_00029 and the three target genes TERG_08278, TERG_02753 and TERG_01198 were all down-regulated in conidia vs. mycelia, while the miRNA Tru-miR3113-3p was significantly up-regulated. These results together indicate that the circRNA and the target genes are positively associated with each other but that they 

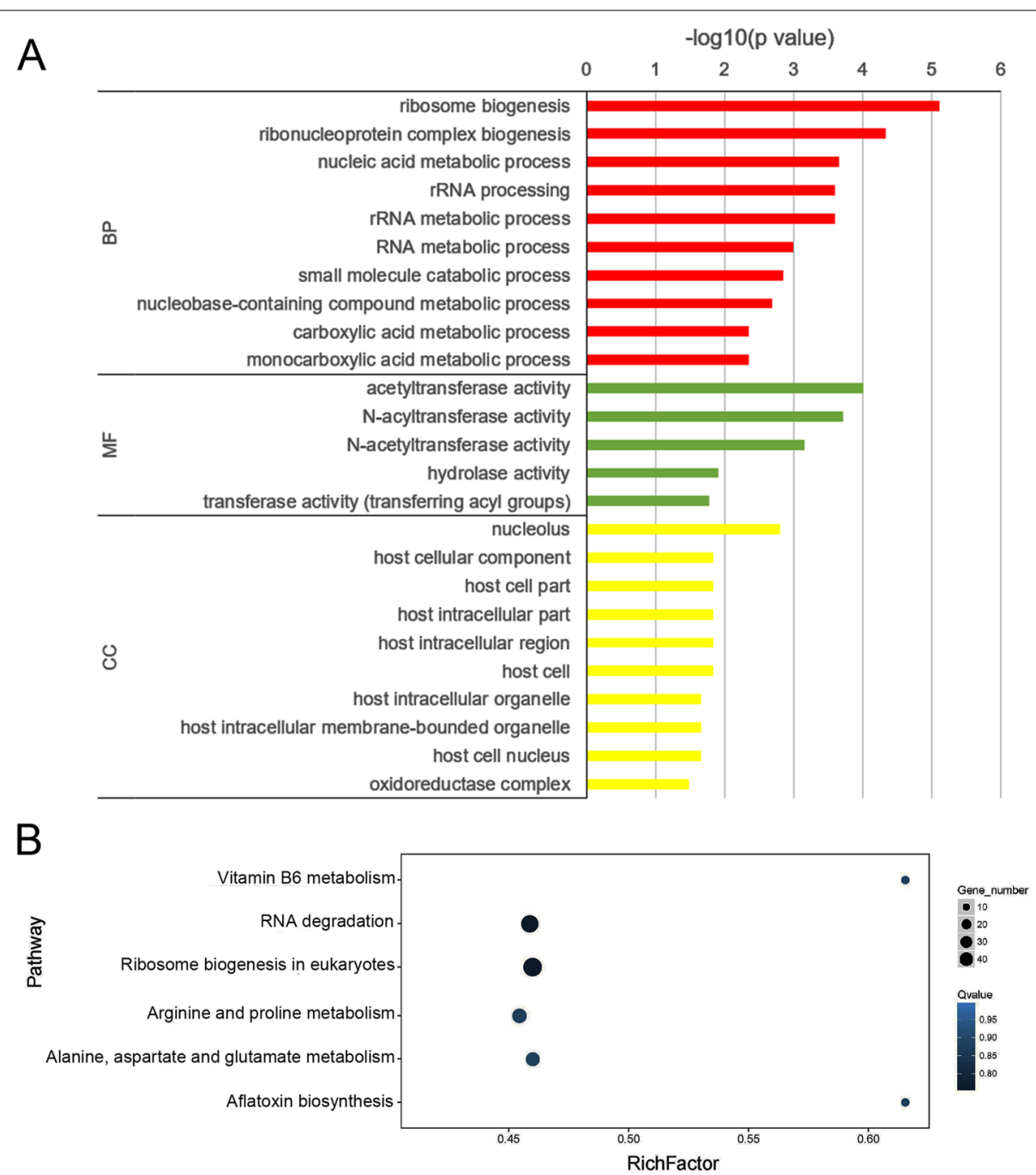

Fig. 7 Functional analysis of target genes for DE-circRNAs. a GO enrichment of target genes based on biological processes (BP), molecular function (MF) and cellular component (CC). b KEGG analysis of target genes

exhibit an expression trend opposite that of the corresponding miRNA. This finding is consistent with the theory that circRNAs competitively sponge miRNAs to release the corresponding target genes inhibited by miRNAs.

\section{Conservation of circRNAs in other species}

To evaluate circRNA conservation, the circRNAs identified in our study were blasted against circRNAs identified in six animal, plant and nematode model organisms. The results revealed, for example (as shown in Table 3), that 12 circRNAs in T. rubrum were homologous to 14 circRNAs in Mus musculus and that 9 circRNAs in T. rubrum were homologous to 3 circRNAs in Gallus gallus. These results suggest that circRNAs are somewhat conserved between T. rubrum and these model species.
Interestingly, the circRNA Tru_circ07138_001 in T. rubrum was homologous to circUBI in G. gallus, cel_circ_0000387 in C. elegans and ghi_circ_000675 in Gossypium spp., with nucleotide sequence identity values of $81.08,94.30$ and $81.82 \%$, respectively. The host genes TERG_07138 (Gene ID: 10372121), UBI (Gene ID: 395296) and ubq-1 (Gene ID: 175840), corresponding to three of these circRNAs, Tru_circ07138_001, circUBI and cel_circ_0000387, respectively, all belong to the short-lived polyubiquitin-related gene family, which is involved in the ATP-dependent proteolysis of impaired proteins $[22,58]$. In addition, each of these three genes generated only one circRNA. The proteins encoded by these three genes were compared, and the sequence similarity was $95.78 \%$ between T. rubrum and G. gallus and 95.63\% between T. rubrum and C. elegans $(\mathrm{E}<1 \mathrm{e}-5)$ (Fig. 9a). These results reveal high conservation of the 

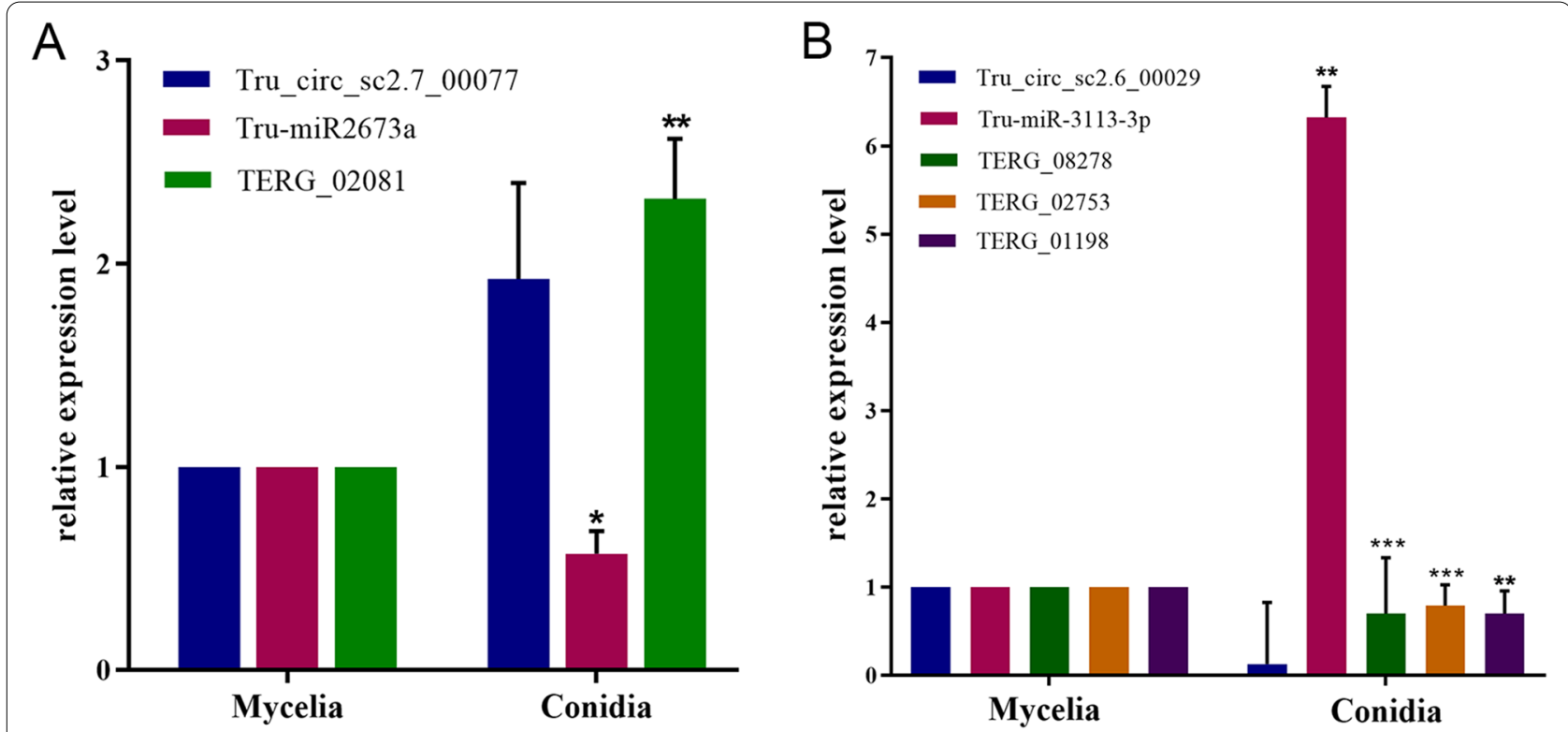

Fig. 8 Relative expression levels of two circRNA-miRNA-mRNA axes between the conidial and mycelial stages. a The axis of circRNA Tru_circ_ sc2.7_00077 - miRNA Tru-miR2673a - target gene TERG_02081. b The axis of the circRNA Tru_circ_sc2.6_00029 - miRNA Tru-miR-3113-3p - target genes TERG_08278, TERG_02753 and TERG_01198. ${ }^{* * *}$ indicates $p<0.001$, ** indicates $p<0.01$ and * indicates $p<0.05$

Table 3 Conservation of T. rubrum circRNAs in six model organisms

\begin{tabular}{llll}
\hline Species & $\begin{array}{l}\text { Number of } \\
\text { circRNAs }\end{array}$ & Blasted species & $\begin{array}{l}\text { Number of } \\
\text { homologous } \\
\text { circRNAs }\end{array}$ \\
\hline T. rubrum & 12 & Mus musculus & 14 \\
T.rubrum & 9 & Gallus gallus & 3 \\
T.rubrum & 5 & Drosophila melanogaster & 6 \\
T.rubrum & 7 & Caenorhabditis elegans & 5 \\
T.rubrum & 14 & Gossypium spp. & 8 \\
T.rubrum & 7 & Arabidopsis thaliana & 1 \\
\hline
\end{tabular}

circRNA Tru_circ07138_001 and its host gene in some distantly related species.

We further explored the conservation of circRNAs between T. rubrum and other dermatophytes. All circRNAs identified in T. rubrum were blasted against the genomes of ten dermatophytes. The results showed that $1752,1710,1689,1635,1564,1578,948,1769,1760$ and 1144 identified circRNAs in T. rubrum were conserved in Trichophyton equinum, Trichophyton tonsurans, Trichophyton mentagrophytes, Trichophyton interdigitale, Trichophyton benhamiae, Trichophyton verrucosum, Trichophyton violaceum, Nannizzia gypsea, Microsporum gypseum and Microsporum canis, respectively (Fig. 9b). Homologous circRNAs accounted for $22-42 \%$ of all circRNAs identified in T. rubrum. Furthermore, 162
circRNAs were conserved in all ten dermatophytes, especially the circRNA Tru_circ07138_001 mentioned above. We annotated and visualized a phylogenetic tree for Tru_circ07138_001 in T. rubrum and the homologous sequences in these ten dermatophytes (Fig. 9c). Among these dermatophyte species, Tru_circ07138_001 was most conserved in T. violaceum, T. benhamiae, T. mentagrophytes and T. interdigitale. However, specificity was also observed. A total of 988 circRNAs were identified only in T. rubrum, suggesting that they play specific roles in this particular species.

\section{Discussion}

CircRNAs are a group of ncRNAs, and their essential roles have been gradually revealed. CircRNAs exhibit specific expression patterns in distinct species, tissues, cell types and developmental stages, so they are considered potential biomarkers for the diagnosis of clinical diseases [8, 59-63]. For example, Li et al. identified abundant circRNAs in human serum exosomes, and these serum exosomal circRNAs can be used to distinguish patients with cancer from healthy controls, suggesting that this type of circRNA can be developed into a promising biomarker for cancer diagnosis [64].

In addition, because circRNAs function as important transcription regulators, investigating the roles of circRNAs will facilitate understanding of the biological and physical features of distinct species [65]. Although the essential roles of circRNAs have been suggested in 


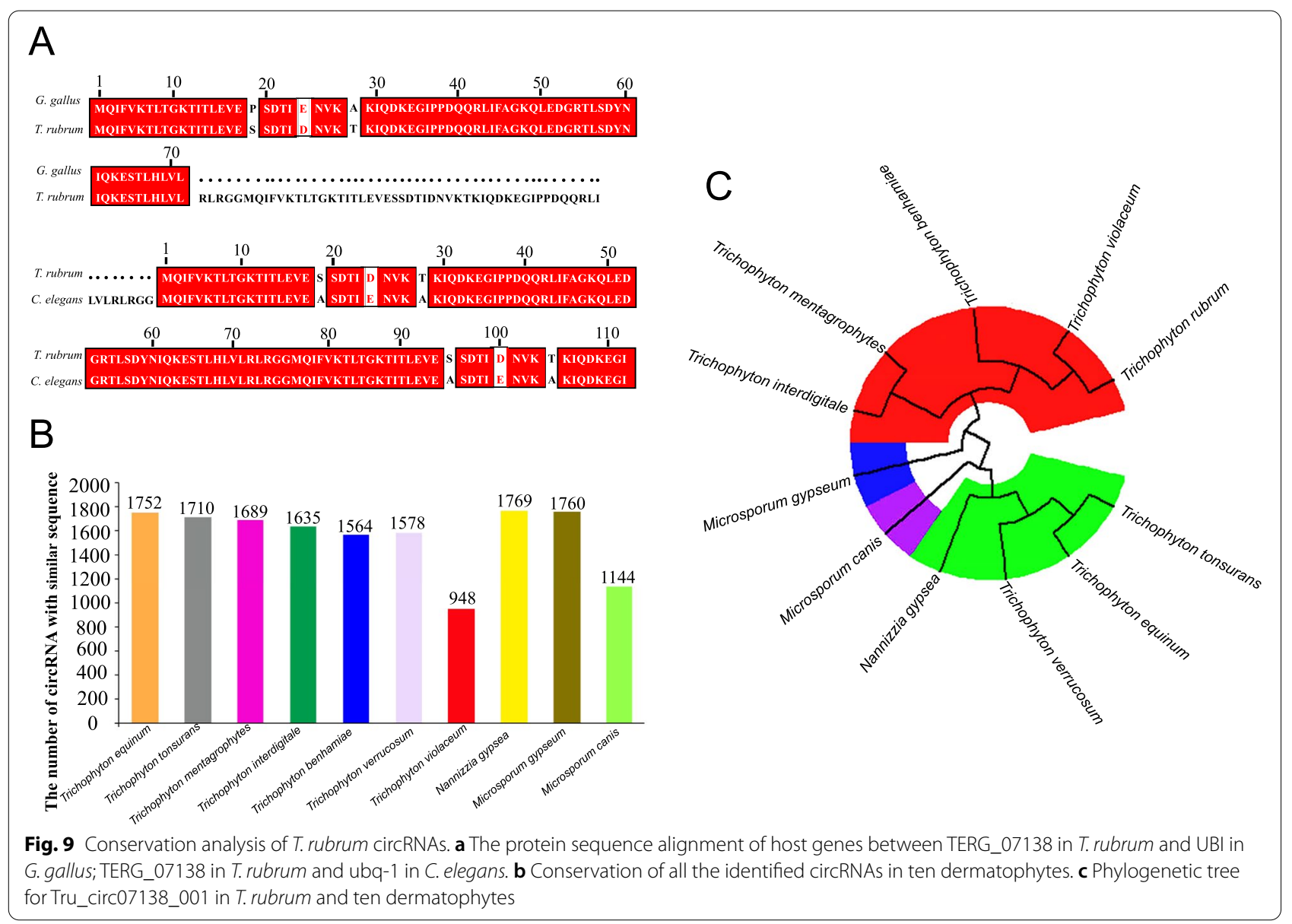

many organisms, the characteristics of fungal circRNAs are largely unknown. T. rubrum is considered a model organism of dermatophytes and human pathogenic filamentous fungi. In our study, circRNAs in T. rubrum were first identified with whole-genome RNA-seq. A total of 4254 circRNAs were identified, and most of them were developmental stage-specific. This pattern is consistent with the results observed in most other species $[28,66]$. Among these, 940 circRNAs were significantly differentially expressed between the two stages. PCR confirmed the circularized structures and relative expression levels of randomly selected circRNAs, supporting the reliability of our data.

Based on the genomic distribution of circRNAs, $60.5 \%$ were derived from intergenic regions, while the others were derived from exonic (35.2\%) and intronic regions (4.3\%). This distribution pattern in T. rubrum is different from those in some animals and plants in which most circRNAs are derived from intronic and exonic regions [67-69]. In contrast, in some fungal species, such as $M$. oryzae and $A$. apis, circRNAs are derived mainly from intergenic regions, displaying distribution patterns similar to those of T. rubrum circRNAs $[27,28]$. The differences in circRNA origin among fungi, animals and plants may be due to the distinct characteristics of circRNAs in different kinds of organisms [70]. In addition, the quality of genome annotation might be an important factor influencing the results of circRNA location. For instance, erroneously divided genes and missing genes in the annotation would increase the proportion of circRNAs in the intergenic regions. Whether the difference in circRNA distribution between T. rubrum and animals and plants is affected by the current computational approach-based annotation would be elucidated with further improved annotation of the T. rubrum genome.

Among all the identified circRNAs, 1680 circRNAs mapped to 1055 host genes. In some cases, two or more circRNAs stemmed from one host gene. Some of these instances may have been due to AS events. Four types of circ-AS events corresponding to 101 circRNAs were identified in our study, including ES, IR, A5SS splicing and A3SS splicing events. Among these, A3SS is the most prevalent splicing event, and ES is the rarest. This AS pattern of circRNAs is different from that 
of linear mRNAs, of which IR is the most common AS type in fungi [71-74]. AS of both circRNA and mRNA is regulated by RNA-binding proteins and splicing factors [1, 75-77]. However, it has been proven that these regulating factors in circRNA binding sites are different from those in linear mRNA binding sites [15]. The distinct preference of regulating factors for circRNA and mRNA might be a possible reason for the difference in their splicing patterns. Furthermore, we found that DEcircRNAs derived from identical host genes showed consistent relative expression levels in conidia vs. mycelia. Whether circRNAs derived from common host genes have the same expression trends and whether interactions occur between circRNAs and their host genes need to be investigated further. Some studies have suggested that circRNAs compete with their host pre-mRNAs, but others have suggested that a subclass of circRNAs (exonintron circRNAs) enhance the transcription of their host genes $[78,79]$. The specific relationships between circRNAs and their host genes should be explored further.

It is widely accepted that circRNAs serve a regulatory role by acting as miRNA sponges and further facilitating mRNA expression, thus constructing a complex ceRNA network $[80,81]$. We depicted a regulatory network of DE-circRNAs in which $661 \mathrm{DE}$-circRNAs targeted 140 miRNAs and further regulated 2753 mRNAs. In this network, one circRNA could target multiple miRNAs, and one miRNA might regulate various mRNAs. We randomly selected two circRNA-miRNA-mRNA axes to investigate their expression patterns. The results all showed similar expression trends of circRNAs and mRNAs that were opposite those of miRNAs, supporting the ceRNA theory. Similar results have also been illustrated in other species. For instance, cells of the human monocyte line THP-1 infected by Trichosporon asahii ( $T$. asahii) were compared with uninfected samples, and a differentially expressed ceRNA network was constructed [82]. The relative expression level of a circRNA-miRNAmRNA axis was investigated by RT-qPCR. The results showed that circRNA hsa_circ_0065336 and target gene PTPN11 were both up-regulated after infection with $T$. asahii, whereas miRNA miR-505-3p was down-regulated [82]. In another instance, circRNA hsa_circ_0007874 was down-regulated in hepatocellular carcinoma (HCC) compared with normal controls, and its related ceRNA pathway hsa_circ_0007874-miR-9-CDX2 was tested with luciferase assays [83]. The results showed that the luciferase activity with the target gene CDX2 decreased dramatically with the increase of miRNA miR-9. However, after circRNA hsa_circ_0007874 was overexpressed, the decline in luciferase activity was repressed [83]. All of these results are consistent with the ceRNA theory, suggesting that circRANs could indirectly regulate target gene expression by sponging miRNAs [82].

Other regulatory factors, such as TFs, may also be involved in the regulation of gene expression based on a ceRNA regulating mechanism [84]. A miRNA could target a TF, and a TF binds to the specific sequence of the enhancer or promoter region of the DNA, thereby suppressing or activating target gene expression in the transcriptional process $[84,85]$. For instance, in spinal cord injury, miRNA miR-674-5p targets a TF Stat1, which regulates the expression of many target genes [86]. Similarly, in our study, miRNA in the ceRNA network was also suggested to target TFs. These processes indicate the complexity of the network that regulates gene expression.

Functional annotation of the target genes suggested that some of them are involved in various small-molecule metabolic processes. Moreover, the target genes were found to be heavily involved in biogenesis-, processing- and metabolism-related processes of ribosomes and RNA, suggesting that these target genes might participate in posttranscriptional processes and protein synthesis. Based on these findings, we speculate that circRNAs might interact with these target genes to affect gene expression at the posttranscriptional and translational levels in addition to acting as miRNA sponges to release mRNA, thus facilitating protein expression. Furthermore, some target genes were identified as secreted proteases and multidrug efflux transporters, suggesting that circRNAs might play a role in the virulence of this fungus.

CircRNAs are highly conserved in mammals [87]. Approximately $25-40 \%$ of circRNAs in humans are homologous to corresponding genomic regions in mice [88]. In addition, $29 \%$ of zebrafish circRNAs show homology with human and mouse circRNAs [21]. In plants, 40 and $8 \%$ of circRNAs identified in A. thaliana are orthologous to circRNAs in Oryza sativa and Solanum lycopersicum, respectively [89]. In our study, we found that circRNAs in T. rubrum exhibited rare conservation with circRNAs in some animal, plant and nematode model organisms that fewer than $1 \%$ of the circRNAs were conserved in these species. This low conservation may be due to the distant phylogenetic relationships between $T$. rubrum and these other species. In contrast, circRNAs in T. rubrum were relatively highly homologous to the corresponding genomic region in closely related dermatophytes, accounting for $22-42 \%$ of all identified circRNAs. In particular, the circRNA Tru_circ07138_001 was conserved in all dermatophyte species analyzed in our study. In addition, this circRNA was conserved in three distantly related species G. gallus, C. elegans and Gossypium spp., and its host gene TERG_07138 was highly homologous to the corresponding circRNA host genes in G. gallus and C. elegans. Interestingly, these three homologous 
host genes all belong to the polyubiquitin-related family. All of these results demonstrate high conservation of this circRNA and its host gene in these different species, suggesting that further exploration of the potential function and role of this conserved circRNA is warranted.

\section{Conclusions}

Our research provides the first global profile of circRNAs in T. rubrum and dermatophytes. These data could lay a foundation for further study on circRNA regulatory mechanisms and provide valuable clues for indepth exploration of the biological characteristics of $T$. rubrum and closely related species. Such pursuits will facilitate the fight against these significant human fungal pathogens.

\section{Methods}

\section{Strain culture and sample collection}

T. rubrum strain BMU 01672 was incubated on potato dextrose agar medium (Becton Dickinson, Sparks, MD, USA) at $28^{\circ} \mathrm{C}$ for 15 days to induce conidia formation. Conidia were collected with distilled water at $4{ }^{\circ} \mathrm{C}$ and then filtered twice through Miracloth (Merck, Billerica, MA, USA) to remove mycelial fragments. Conidial purity was examined with a microscope. To obtain mycelia, $1 \times 10^{6}$ conidia were inoculated into $100 \mathrm{ml}$ YPD liquid medium (Becton Dickinson) and cultured at $28^{\circ} \mathrm{C}$ under constant shaking $(180 \mathrm{rpm})$ for $72 \mathrm{~h}$ [90]. The medium was removed by thorough washing with cold distilled water, and mycelia were collected through centrifugation at $4000 \times g$ for $5 \mathrm{~min}$.

\section{RNA extraction and library sequencing}

Three biological replicates were performed for each conidial and mycelial stage, and $300 \mathrm{mg}$ material was used for each replicate. Total RNA was extracted using TRIzol reagent (Invitrogen, Carlsbad, CA, USA) according to the manufacturer's protocol. Then, RNase-free DNase (Qiagen, Hilden, Germany) was added to eliminate potential genomic DNA contamination. The integrity and purity of total RNA were assessed with an Agilent 2100 Bioanalyzer system (Agilent Technologies, Santa Clara, CA, USA) and a NanoDrop ND-1000 spectrophotometer (Thermo Scientific, Waltham, MA, USA).

In each sample, rRNA was removed using a Ribo-Zero rRNA Removal Kit (Epicentre, Madison, WI, USA), and linear RNA was digested with RNase R (Epicentre). The remaining RNA was fragmented, and the fragments were reverse-transcribed to construct cDNA libraries following the protocol of an NEBNext Ultra Directional RNA Library Prep Kit for Illumina (NEB, Ipswich, MA, USA). After repairing and filling the ends of double-stranded cDNA, NEBNext adaptors were ligated. cDNA fragments of 150-200 bp were selected and purified using AMPure XP Beads (Beckman Coulter, Beverly, MA, USA). Afterward, the ligated products were PCR-amplified, and the library quality and quantity were evaluated with an Agilent Bioanalyzer 2100 system and qRT-PCR. Finally, $150 \mathrm{bp}$ paired-end reads were sequenced on the Illumina HiSeq $^{\text {TM }} 4000$ platform.

\section{CircRNA identification and differential expression analysis} Adaptors and low-quality reads that contained poly- $\mathrm{N}$ sequences or had low $\mathrm{Q}$ scores were removed to obtain clean reads, and the Q20, Q30, GC content and sequence duplication level were calculated. The clean reads were mapped to the T. rubrum reference genome using BWA software to identify junction reads [91]. These junction reads were realigned to the T. rubrum reference genome to determine the splice sites. The junction reads with paired chiastic clipping (PCC) signals and paired-end mapping (PEM) sites were considered high-confidence back-spliced junction reads. In addition, only the backspliced junction reads flanking the GU/AG splice signal were considered further. CircRNAs were identified using the CIRI tool based on a cutoff value of back-spliced junction reads $\geq 2$ [9]. We also used CircRNA IdentifierAlternative Splicing (CIRI-AS) software to identify AS events in circRNAs [15].

The spliced reads per billion mapping (SRPBM) values were calculated to normalize the circRNA reads in each sample, and the number of junction reads per circRNA was determined with Circos software. The differential expression values of circRNAs were calculated using NOIseq, and DE-circRNAs were identified with the thresholds of $\mid \log _{2}$ (fold change) $\mid \geq 1$ and probability $\geq 0.8$ $[92,93]$.

\section{Gene function annotation and ceRNA network construction}

Gene function annotation was performed based on GO terms using the clusterProfiler R package [94] and KEGG (https://www.kegg.jp/) [95-97]. MiRNA target sites of circRNAs were predicted with the miRanda program based on the conserved seed-matching sequence [98], and mRNAs targeted by miRNAs were identified using TargetFinder software [99]. Based on the theory of ceRNA, we constructed a circRNA-miRNA-mRNA interaction network using Cytoscape 3.5.1 software [100].

\section{Conservation analysis of $T$. rubrum circRNAs}

Conservation of circRNAs was investigated in six distantly related species and ten closely related dermatophytes. CircRNAs identified in T. rubrum were blasted against circRNAs of six distantly related species, including G. gallus, D. melanogaster, Gossypium spp., A. 
thaliana, C. elegans, and M. musculus, using BlastN with the criteria of score $>50$, identity $>50 \%$, and $E$ value $<1 \mathrm{e}^{-5}$. The circRNAs of these six species were deposited in the circBase database (http://www.circbase.org/) and the CircFunBase database (http://bis.zju.edu.cn/ CircFunBaseBlast/). Genome sequences of ten dermatophytes, including T. equinum CBS 127.97, T. tonsurans CBS 112818, T. mentagrophytes D15P127, T. interdigitale MR816, T. benhamiae CBS 112371, T. verrucosum HKI 0517, T. violaceum CMCC (F) T31, N. gypsea CBS 118893, M. gypseum CBS 118893 and M. canis CBS 113480, were downloaded from NCBI (https://www.ncbi. nlm.nih.gov/). The genome sequences of all circRNAs identified in T. rubrum were then compared with these dermatophyte genomes using BlastN (v2.11.0, $\mathrm{E}<1 \mathrm{e}^{-5}$ ). A phylogenetic tree of circRNAs was visualized and annotated using the ggtree R package [101]. Multiple sequence alignments were conducted using the ClustalW web server with the default settings [102].

\section{Validation of circRNA circularization by PCR and Sanger sequencing}

Total RNA was treated with DNase I and RNase R, and the remaining RNA was reverse-transcribed into cDNA with random primers using a SuperScript ${ }^{\circledR}$ III FirstStrand Synthesis System (Invitrogen) following the manufacturer's protocol. Genomic DNA was extracted using a DNeasy Plant Mini Kit (Qiagen). Divergent primers and convergent primers of seven randomly selected DEcircRNAs were designed using Primer-BLAST (http:// www.ncbi.nlm.nih.gov/tools/primer-blast) according to the methods in previous studies $[68,87]$. All primer sequences are provided in Additional file 14: Table S13. The PCR procedure was as follows: $94^{\circ} \mathrm{C}$ for $1 \mathrm{~min}$; 35 cycles of $98^{\circ} \mathrm{C}$ for $10 \mathrm{~s}$, annealing at $52^{\circ} \mathrm{C}$ for $30 \mathrm{~s}$ and extension at $72{ }^{\circ} \mathrm{C}$ for $12 \mathrm{~s}$; and a final extension at $72^{\circ} \mathrm{C}$ for $10 \mathrm{~min}$. The PCR products were detected using $2 \%$ agarose gel electrophoresis. The expected bands were excised, and the DNA was eluted using a QIAquick gel extraction kit (Qiagen). Sanger sequencing was performed to further verify the existence of the back-spliced junction sites of these circRNAs.

\section{Validation of circRNA relative expression levels}

The relative expression levels of the seven DE-circRNAs illustrated above were validated by qRT-PCR with divergent primers (Additional file 14: Table S13). qRT-PCR was performed on an ABI 7500 Real-Time PCR Detection System (Applied Biosystems, Waltham, MA, USA) using PowerUp ${ }^{\mathrm{TM}}$ SYBR $^{\mathrm{TM}}$ Green Master Mix (Applied Biosystems). The conditions were as follows: initial denaturation for $2 \mathrm{~min}$ at $95^{\circ} \mathrm{C}$ followed by 40 cycles of $15 \mathrm{~s}$ at $95^{\circ} \mathrm{C}, 15 \mathrm{~s}$ at $58^{\circ} \mathrm{C}$, and $60 \mathrm{~s}$ extension at $72^{\circ} \mathrm{C}$. The relative expression level was calculated using the $2^{-\Delta \Delta \mathrm{Ct}}$ method [103]. Data were normalized with two reference genes, DNA-dependent RNA polymerase II (rpb2) and chitin synthase (chs1), as previously described [104]. Statistical significance was determined with GraphPad Prism 8 software (GraphPad Software Inc., San Diego, CA, USA). $p<0.05$ was considered to indicate significance.

\section{Detection of the relative expression levels of two circRNA-miRNA-mRNA axes}

We randomly selected two circRNA-miRNA-mRNA axes and examined the relative expression levels of the circRNAs, miRNAs and mRNAs in these axes with respect to the conidial and mycelial stages. The primer sequences are listed in Additional file 14: Table S13. qRT-PCR of mRNA was performed with reagents and procedures identical to those described above for circRNAs. The procedure for miRNA extraction was as follows: total RNA was extracted using a miRNeasy Mini Kit (Qiagen), and gDNA was eliminated using a TURBO DNAfree ${ }^{\mathrm{TM}} \mathrm{Kit}$ (Thermo Fisher Scientific) at $37^{\circ} \mathrm{C}$ for $30 \mathrm{~min}$. MiRNA was then reverse-transcribed with a miRcute Plus miRNA First-Strand cDNA kit (TIANGEN, Beijing, China) in a $20 \mu \mathrm{l}$ reaction volume at $42^{\circ} \mathrm{C}$ for $60 \mathrm{~min}$ and $95^{\circ} \mathrm{C}$ for $3 \mathrm{~min}$. qRT-PCR of miRNA was performed using miRcute SYBR Green Master Mix (TIANGEN). The reaction conditions were $15 \mathrm{~min}$ at $95^{\circ} \mathrm{C}$ followed by 40 cycles of $20 \mathrm{~s}$ at $94^{\circ} \mathrm{C}$ and $34 \mathrm{~s}$ at $60^{\circ} \mathrm{C}$. U6 was used as the internal control of miRNAs for gene expression normalization. Each experiment was performed with three replicates. The relative expression level and statistical significance were calculated as stated above.

\section{Abbreviations}

A3SS: Alternative 3' splicing site; A5SS: Alternative 5' splicing site; AS: Alternative splicing; CircRNA: Circular RNA; circ-AS: Circular RNA alternative splicing; ceRNA: Competing endogenous RNA; DE-circRNA: Differentially expressed circRNA; IR: Intron retention; miRNA: microRNA; ncRNA: Non-coding RNA; pre-mRNA: Precursor mRNA; RNA-seq: High-throughput RNA sequencing; SE: Skipping exon; SRPBM: Spliced reads per billion mapping; TFs: Transcription factors; T. rubrum: Trichophyton rubrum.

\section{Supplementary Information}

The online version contains supplementary material available at https://doi. org/10.1186/s12864-021-08184-y.

Additional file 1: Figure S1-S8. All supplementary figures.

Additional file 2: Table S1. CircRNAs identified in conidial and mycelial stage of T. rubrum.

Additional file 3: Table S2. Significant differentially expressed circRNAs in conidial vs. mycelial stage.

Additional file 4: Table S3. GO classification of host genes for all the identified circRNAs.

Additional file 5: Table S4. KEGG pathway analysis of host genes for all the identified circRNAs. 

Additional file 6: Table S5. GO classification of host genes for
DE-circRNAs.

Additional file 7: Table S6. KEGG pathways analysis of host genes for DE-circRNAs.

Additional file 8: Table S7. CircRNA AS events identified in T. rubrum conidial and mycelial stages.

Additional file 9: Table S8. MiRNAs targeted by DE-circRNAs.

Additional file 10: Table S9. mRNAs targeted by miRNAs.

Additional file 11: Table S10. GO enrichment of target genes for DE-circRNAs.

Additional file 12: Table S11. KEGG pathways analysis of target genes for DE-circRNAs.

Additional file 13: Table S12. The target genes related to fungal pathogenicity.

Additional file 14: Table S13. Primers used in this study.

\section{Acknowledgments}

Not applicable.

\section{Authors' contributions}

$T L$ and QJ designed and supervised the research. XC and XX performed experiments and wrote the manuscript. JD, YX, LS and $Y Z$ participated in the experiments. All authors read and approved the final manuscript.

\section{Funding}

This work was financially supported by the CAMS Innovation Fund for Medical Sciences (CIFMS) (Grant No. 2021-I2M-1-038) and the National Science and Technology Major Project of China (Grant no. 2018ZX10712001-002-001, 2018ZX10711001). The funder had no role in the study design, data collection and analysis or preparation of the manuscript.

\section{Availability of data and materials}

The data supporting the conclusions of this article are within the paper and its additional files. The sequence data generated from T. rubrum conidia and mycelia has been deposited in the NCBI SRA database with the accession number PRJNA701864, which can be accessed with https://www.ncbi.nlm.nih. gov//bioproject/PRJNA701864.

\section{Declarations}

\section{Ethics approval and consent to participate}

Not applicable.

\section{Consent for publication}

Not applicable.

\section{Competing interests}

The authors declare that they have no competing interests.

Received: 5 March 2021 Accepted: 18 November 2021

Published online: 04 January 2022

\section{References}

1. Ashwal-Fluss R, Meyer M, Pamudurti NR, Ivanov A, Bartok O, Hanan M, et al. circRNA biogenesis competes with pre-mRNA splicing. Mol Cell. 2014;56(1):55-66.

2. Qu S, Yang X, Li X, Wang J, Gao Y, Shang R, et al. Circular RNA: a new star of noncoding RNAs. Cancer Lett. 2015;365(2):141-8.

3. Sanger HL, Klotz G, Riesner D, Gross HJ, Kleinschmidt AK. Viroids are single-stranded covalently closed circular RNA molecules existing as highly base-paired rod-like structures. Proc Natl Acad Sci U S A. 1976;73(11):3852-6.
4. Kos A, Dijkema R, Arnberg AC, van der Meide PH, Schellekens H. The hepatitis delta (delta) virus possesses a circular RNA. Nature. 1986:323(6088):558-60.

5. Nigro JM, Cho KR, Fearon ER, Kern SE, Ruppert JM, Oliner JD, et al. Scrambled exons. Cell. 1991;64(3):607-13.

6. Cocquerelle C, Mascrez B, Hétuin D, Bailleul B. Mis-splicing yields circular RNA molecules. FASEB J. 1993;7(1):155-60.

7. Dudekula DB, Panda AC, Grammatikakis I, De S, Abdelmohsen K, Gorospe M. Circlnteractome: a web tool for exploring circular RNAs and their interacting proteins and microRNAs. RNA Biol. 2016;13(1):34-42.

8. Westholm JO, Miura P, Olson S, Shenker S, Joseph B, Sanfilippo P, et al. Genome-wide analysis of drosophila circular RNAs reveals their structural and sequence properties and age-dependent neural accumulation. Cell Rep. 2014;9(5):1966-80.

9. Gao Y, Wang J, Zhao F. CIRI: an efficient and unbiased algorithm for de novo circular RNA identification. Genome Biol. 2015;16(1):4.

10. Memczak S, Jens M, Elefsinioti A, Torti F, Krueger J, Rybak A, et al. Circular RNAs are a large class of animal RNAs with regulatory potency. Nature. 2013;495(7441):333-8.

11. Zhang XO, Wang HB, Zhang Y, Lu X, Chen LL, Yang L. Complementary sequence-mediated exon circularization. Cell. 2014;159(1):134-47.

12. Liu YC, Li JR, Sun CH, Andrews E, Chao RF, Lin FM, et al. CircNet: a database of circular RNAs derived from transcriptome sequencing data. Nucleic Acids Res. 2016;44(D1):D209-15.

13. Ghosal S, Das S, Sen R, Basak P, Chakrabarti J. Circ2Traits: a comprehensive database for circular RNA potentially associated with disease and traits. Front Genet. 2013;4:283.

14. Zheng LL, Li JH, Wu J, Sun WJ, Liu S, Wang ZL, et al. deepBase v2.0: identification, expression, evolution and function of small RNAs, LncRNAs and circular RNAs from deep-sequencing data. Nucleic Acids Res. 2016;44(D1):D196-202.

15. Gao Y, Wang J, Zheng Y, Zhang J, Chen S, Zhao F. Comprehensive identification of internal structure and alternative splicing events in circular RNAs. Nat Commun. 2016;7:12060.

16. Suzuki H, Tsukahara T. A view of pre-mRNA splicing from RNase R resistant RNAs. Int J Mol Sci. 2014;15(6):9331-42.

17. Hansen TB, Jensen TI, Clausen BH, Bramsen JB, Finsen B, Damgaard CK, et al. Natural RNA circles function as efficient microRNA sponges. Nature. 2013;495(7441):384-8

18. Yang $Y$, Fan $X$, Mao M, Song X, Wu P, Zhang Y, et al. Extensive translation of circular RNAs driven by N (6)-methyladenosine. Cell Res. 2017;27(5):626-41.

19. Salzman J, Gawad C, Wang PL, Lacayo N, Brown PO. Circular RNAs are the predominant transcript isoform from hundreds of human genes in diverse cell types. PLoS One. 2012;7(2):e30733.

20. Zhou Z, Du D, Chen A, Zhu L. Circular RNA expression profile of articular chondrocytes in an IL-1 $\beta$-induced mouse model of osteoarthritis. Gene. 2018;644:20-6.

21. Shen $Y$, Guo $X$, Wang W. Identification and characterization of circular RNAs in zebrafish. FEBS Lett. 2017;591(1):213-20.

22. Cortés-López M, Gruner MR, Cooper DA, Gruner HN, Voda Al, van der Linden AM, et al. Global accumulation of circRNAs during aging in Caenorhabditis elegans. BMC Genomics. 2018;19(1):8.

23. Wang PL, Bao Y, Yee MC, Barrett SP, Hogan GJ, Olsen MN, et al. Circular RNA is expressed across the eukaryotic tree of life. PLoS One. 2014;9(6):e90859.

24. Zhang J, Liu R, Zhu Y, Gong J, Yin S, Sun P, et al. Identification and characterization of circRNAs responsive to methyl Jasmonate in Arabidopsis thaliana. Int J Mol Sci. 2020;21(3):792.

25. Lu T, Cui L, Zhou Y, Zhu C, Fan D, Gong H, et al. Transcriptome-wide investigation of circular RNAs in rice. RNA. 2015;21(12):2076-87.

26. Lv L, Yu K, Lü H, Zhang X, Liu X, Sun C, et al. Transcriptome-wide identification of novel circular RNAs in soybean in response to low-phosphorus stress. PLoS One. 2020;15(1):e0227243.

27. Guo R, Chen D, Chen H, Fu Z, Xiong C, Hou C, et al. Systematic investigation of circular RNAs in Ascosphaera apis, a fungal pathogen of honeybee larvae. Gene. 2018;678:17-22.

28. Yuan J, Wang Z, Xing J, Yang Q, Chen XL. Genome-wide identification and characterization of circular RNAs in the rice blast fungus Magnaporthe oryzae. Sci Rep. 2018;8(1):6757. 
29. Guo R, Chen D, Chen H, Xiong C, Zheng Y, Hou C, et al. Genome-wide identification of circular RNAs in fungal parasite Nosema ceranae. Curr Microbiol. 2018;75(12):1655-60.

30. Aly R. Ecology and epidemiology of dermatophyte infections. J Am Acad Dermatol. 1994;31(3 Pt 2):S21-5.

31. Faway E, Cambier L, De Vuyst E, Evrard C, Thiry M, Lambert de Rouvroit C, et al. Responses of reconstructed human epidermis to Trichophyton rubrum infection and impairment of infection by the inhibitor PD169316. J Invest Dermatol. 2019;139(10):2080-89.e6.

32. Liu T, Zhang Q, Wang L, Yu L, Leng W, Yang J, et al. The use of global transcriptional analysis to reveal the biological and cellular events involved in distinct development phases of Trichophyton rubrum conidial germination. BMC Genomics. 2007;8:100.

33. Kuklová I, Stork J, Lacina L. Trichophyton rubrum suppurative tinea of the bald area of the scalp. Mycoses. 2011;54(1):84-6.

34. Rodwell GE, Bayles CL, Towersey L, Aly R. The prevalence of dermatophyte infection in patients infected with human immunodeficiency virus. Int J Dermatol. 2008;47(4):339-43.

35. Lee WJ, Kim SL, Jang YH, Lee SJ, Kim DW, Bang YJ, et al. Increasing prevalence of Trichophyton rubrum identified through an analysis of 115,846 cases over the last 37 years. J Korean Med Sci. 2015;30(5):639-43.

36. Zheng H, Blechert O, Mei H, Ge L, Liu J, Tao Y, et al. Whole-genome resequencing of Trichophyton rubrum provides insights into population differentiation and drug resistance. Mycopathologia. 2020;185(1):103-12.

37. Bitencourt TA, Macedo C, Franco ME, Assis AF, Komoto TT, Stehling EG, et al. Transcription profile of Trichophyton rubrum conidia grown on keratin reveals the induction of an adhesin-like protein gene with a tandem repeat pattern. BMC Genomics. 2016;17:249.

38. Bitencourt TA, Macedo C, Franco ME, Rocha MC, Moreli IS, Cantelli BAM, et al. Trans-chalcone activity against Trichophyton rubrum relies on an interplay between signaling pathways related to cell wall integrity and fatty acid metabolism. BMC Genomics. 2019;20(1):411.

39. Martinez DA, Oliver BG, Graser Y, Goldberg JM, Li W, Martinez-Rossi NM, et al. Comparative genome analysis of Trichophyton rubrum and related dermatophytes reveals candidate genes involved in infection. MBio. 2012;3(5):e00259-12.

40. Liu T, Ren X, Xiao T, Yang J, Xu X, Dong J, et al. Identification and characterisation of non-coding small RNAs in the pathogenic filamentous fungus Trichophyton rubrum. BMC Genomics. 2013;14:931.

41. Xu X, Cao X, Yang J, Chen L, Liu B, Liu T, et al. Proteome-wide identification of lysine Propionylation in the conidial and mycelial stages of Trichophyton rubrum. Front Microbiol. 2019;10:2613.

42. Xu X, Liu T, Yang J, Chen L, Liu B, Wang L, et al. The first whole-cell proteome- and lysine-Acetylome-based comparison between Trichophyton rubrum conidial and mycelial stages. J Proteome Res. 2018;17(4):1436-51.

43. Xu X, Liu T, Yang J, Chen L, Liu B, Wei C, et al. The first succinylome profile of Trichophyton rubrum reveals lysine succinylation on proteins involved in various key cellular processes. BMC Genomics. 2017;18(1):577.

44. Zhang L, Feng Q, Sun L, Ding K, Huo D, Fang Y, et al. Differential gene expression in the intestine of sea cucumber (Apostichopus japonicus) under low and high salinity conditions. Comp Biochem Physiol Part D Genomics Proteomics. 2018;25:34-41.

45. Diaz-Riano J, Posada L, Acosta IC, Ruiz-Perez C, Garcia-Castillo C, Reyes A, et al. Computational search for UV radiation resistance strategies in Deinococcus swuensis isolated from Paramo ecosystems. PLoS One. 2019;14(12):e0221540.

46. Zhang Y, Nyong AT, Shi T, Yang P. The complexity of alternative splicing and landscape of tissue-specific expression in lotus (Nelumbo nucifera) unveiled by Illumina- and single-molecule real-time-based RNAsequencing. DNA Res. 2019;26(4):301-11.

47. Chuang TJ, Chen YJ, Chen CY, Mai TL, Wang YD, Yeh CS, et al. Integrative transcriptome sequencing reveals extensive alternative trans-splicing and cis-backsplicing in human cells. Nucleic Acids Res. 2018:46(7):3671-91.

48. Wang $\mathrm{H}$, Wang H, Zhang H, Liu S, Wang Y, Gao Y, et al. The interplay between microRNA and alternative splicing of linear and circular RNAs in eleven plant species. Bioinformatics. 2019;35(17):3119-26.
49. Zhou B, Yu JW. A novel identified circular RNA, circRNA_010567, promotes myocardial fibrosis via suppressing miR-141 by targeting TGF- $\beta 1$. Biochem Biophys Res Commun. 2017;487(4):769-75.

50. Zhang S, Zhu D, Li H, Li H, Feng C, Zhang W. Characterization of circRNA-associated-ceRNA networks in a senescence-accelerated mouse prone 8 brain. Mol Ther. 2017;25(9):2053-61.

51. Thölken C, Thamm M, Erbacher C, Lechner M. Sequence and structural properties of circular RNAs in the brain of nurse and forager honeybees (Apis mellifera). BMC Genomics. 2019;20(1):88.

52. Wang L, Xu X, Yang J, Chen L, Liu B, Liu T, et al. Integrated microRNA and mRNA analysis in the pathogenic filamentous fungus Trichophyton rubrum. BMC Genomics. 2018;19(1):933.

53. Sriranganadane D, Waridel P, Salamin K, Feuermann M, Mignon $B$, Staib P, et al. Identification of novel secreted proteases during extracellular proteolysis by dermatophytes at acidic $\mathrm{pH}$. Proteomics. 2011;11(22):4422-33.

54. Mercer DK, Stewart CS. Keratin hydrolysis by dermatophytes. Med Mycol. 2019;57(1):13-22.

55. Monod M. Secreted proteases from dermatophytes. Mycopathologia. 2008;166(5-6):285-94.

56. Cannon RD, Lamping E, Holmes AR, Niimi K, Baret PV, Keniya MV, et al. Efflux-mediated antifungal drug resistance. Clin Microbiol Rev. 2009;22(2):291-321 Table of Contents.

57. Costa C, Dias PJ, Sa-Correia I, Teixeira MC. MFS multidrug transporters in pathogenic fungi: do they have real clinical impact? Front Physiol. 2014;5:197.

58. Zhang X, Yan Y, Lei X, Li A, Zhang H, Dai Z, et al. Circular RNA alterations are involved in resistance to avian leukosis virus subgroup-J-induced tumor formation in chickens. Oncotarget. 2017:8(21):34961-70.

59. Wang Z, Liu Y, Li D, Li L, Zhang Q, Wang S, et al. Identification of circular RNAs in kiwifruit and their species-specific response to bacterial canker pathogen invasion. Front Plant Sci. 2017;8:413.

60. Xia S, Feng J, Lei L, Hu J, Xia L, Wang J, et al. Comprehensive characterization of tissue-specific circular RNAs in the human and mouse genomes. Brief Bioinform. 2017;18(6):984-92.

61. Salzman J, Chen RE, Olsen MN, Wang PL, Brown PO. Cell-type specific features of circular RNA expression. PLoS Genet. 2013;9(9):e1003777.

62. Li JJ, Wang W, Wang XQ, He Y, Wang SS, Yan YX. A novel strategy of identifying circRNA biomarkers in cardiovascular disease by meta-analysis. J Cell Physiol. 2019;234(12):21601-12.

63. Qin M, Liu G, Huo X, Tao X, Sun X, Ge Z, et al. Hsa_circ_0001649: a circular RNA and potential novel biomarker for hepatocellular carcinoma. Cancer Biomark. 2016;16(1):161-9.

64. Li Y, Zheng Q, Bao C, Li S, Guo W, Zhao J, et al. Circular RNA is enriched and stable in exosomes: a promising biomarker for cancer diagnosis. Cell Res. 2015;25(8):981-4.

65. Li Z, Huang C, Bao C, Chen L, Lin M, Wang X, et al. Exon-intron circular RNAs regulate transcription in the nucleus. Nat Struct Mol Biol. 2015;22(3):256-64.

66. Cai Y, Lei $X, C$ Chen Z, Mo Z. The roles of cirRNA in the development of germ cells. Acta Histochem. 2020;122(3):151506.

67. Wang L, You Z, Wang M, Yuan Y, Liu C, Yang N, et al. Genome-wide analysis of circular RNAs involved in Marek's disease tumourigenesis in chickens. RNA Biol. 2020;17(4):517-27.

68. Shen M, LiT, Zhang G, Wu P, Chen F, Lou Q, et al. Dynamic expression and functional analysis of circRNA in granulosa cells during follicular development in chicken. BMC Genomics. 2019;20(1):96.

69. Ye CY, Chen L, Liu C, Zhu QH, Fan L. Widespread noncoding circular RNAs in plants. New Phytol. 2015;208(1):88-95.

70. Sun LF, Zhang B, Chen XJ, Wang XY, Zhang BW, Ji YY, et al. Circular RNAs in human and vertebrate neural retinas. RNA Biol. 2019;16(6):821-9.

71. Neves-da-Rocha J, Bitencourt TA, Oliveira VM, Sanches PR, Rossi A, Martinez-Rossi NM. Alternative splicing in heat shock protein transcripts as a mechanism of cell adaptation in Trichophyton rubrum. Cells. 2019;8(10):1206.

72. Grutzmann K, Szafranski K, Pohl M, Voigt K, Petzold A, Schuster S. Fungal alternative splicing is associated with multicellular complexity and virulence: a genome-wide multi-species study. DNA Res. 2014;21(1):27-39.

73. Kempken F. Alternative splicing in ascomycetes. Appl Microbiol Biotechnol. 2013;97(10):4235-41. 
74. Gomes EV, Bortolossi JC, Sanches PR, Mendes NS, Martinez-Rossi NM, Rossi A. STE20/PAKA protein kinase gene releases an autoinhibitory domain through pre-mRNA alternative splicing in the dermatophyte Trichophyton rubrum. Int J Mol Sci. 2018;19(11):3654.

75. Conn SJ, Pillman KA, Toubia J, Conn VM, Salmanidis M, Phillips CA, et al. The RNA binding protein quaking regulates formation of circRNAs. Cell. 2015;160(6):1125-34.

76. Fu XD, Ares M Jr. Context-dependent control of alternative splicing by RNA-binding proteins. Nat Rev Genet. 2014;15(10):689-701.

77. McManus CJ, Graveley BR. RNA structure and the mechanisms of alternative splicing. Curr Opin Genet Dev. 2011;21(4):373-9.

78. Zhang L, Liu X, Che S, Cui J, Liu Y, An X, et al. CircRNA-9119 regulates the expression of prostaglandin-endoperoxide synthase 2 (PTGS2) by sponging miR-26a in the endometrial epithelial cells of dairy goat. Reprod Fertil Dev. 2018;30(12):1759-69.

79. Tang CM, Zhang M, Huang L, Hu ZQ, Zhu JN, Xiao Z, et al. CirCRNA_000203 enhances the expression of fibrosis-associated genes by derepressing targets of miR-26b-5p, Col1a2 and CTGF, in cardiac fibroblasts. Sci Rep. 2017;7:40342.

80. Zhang Y, Zhang H, An M, Zhao B, Ding H, Zhang Z, et al. Crosstalk in competing endogenous RNA networks reveals new circular RNAs involved in the pathogenesis of early HIV infection. J Transl Med. 2018;16(1):332.

81. Huang Q, Huang QY, Sun Y, Wu S. High-throughput data reveals novel circular RNAs via competitive endogenous RNA networks associated with human intracranial aneurysms. Med Sci Monit. 2019;25:4819-30.

82. Zhang MW, Zhu ZH, Xia ZK, Yang X, Luo WT, Ao JH, et al. Comprehensive circRNA-microRNA-mRNA network analysis revealed the novel regulatory mechanism of Trichosporon asahii infection. Mil Med Res. 2021:8(1):19.

83. Li X, Ding J, Wang X, Cheng Z, Zhu Q. NUDT21 regulates circRNA cyclization and ceRNA crosstalk in hepatocellular carcinoma. Oncogene. 2020;39(4):891-904.

84. Liu Y, Xie D, He Z, Zheng L. Integrated analysis reveals five potential ceRNA biomarkers in human lung adenocarcinoma. PeerJ. 2019;7:e6694

85. Cai J, Yu Y, Xu Y, Liu H, Shou J, You L, et al. Exploring the role of Mir204/211 in HNSCC by the combination of bioinformatic analysis of ceRNA and transcription factor regulation. Oral Oncol. 2019;96:153-60.

86. Wang N, He L, Yang Y, Li S, Chen Y, Tian Z, et al. Integrated analysis of competing endogenous RNA (ceRNA) networks in subacute stage of spinal cord injury. Gene. 2020;726:144171.

87. Jeck WR, Sorrentino JA, Wang K, Slevin MK, Burd CE, Liu J, et al. Circular RNAs are abundant, conserved, and associated with ALU repeats. RNA. 2013;19(2):141-57.

88. Legnini I, Di Timoteo G, Rossi F, Morlando M, Briganti F, Sthandier O, et al. Circ-ZNF609 is a circular RNA that can be translated and functions in Myogenesis. Mol Cell. 2017;66(1):22-37.e9.

89. Chen G, Cui J, Wang L, Zhu Y, Lu Z, Jin B. Genome-wide identification of circular RNAs in Arabidopsis thaliana. Front Plant Sci. 2017:8:1678.

90. Lopes L, Bitencourt TA, Lang EAS, Sanches PR, Peres NTA, Rossi A, et al. Genes coding for LysM domains in the dermatophyte Trichophyton rubrum: a transcription analysis. Med Mycol. 2020;58(3):372-9.

91. Li H, Durbin R. Fast and accurate short read alignment with burrowswheeler transform. Bioinformatics. 2009;25(14):1754-60.

92. Tarazona S, García-Alcalde F, Dopazo J, Ferrer A, Conesa A. Differential expression in RNA-seq: a matter of depth. Genome Res. 2011;21(12):2213-23.

93. Tarazona S, Furio-Tari P, Turra D, Pietro AD, Nueda MJ, Ferrer A, et al. Data quality aware analysis of differential expression in RNA-seq with NOISeq R/bioc package. Nucleic Acids Res. 2015;43(21):e140.

94. Yu G, Wang LG, Han Y, He QY. clusterProfiler: an R package for comparing biological themes among gene clusters. OMICS. 2012;16(5):284-7.

95. Kanehisa M, Goto S. KEGG: Kyoto encyclopedia of genes and genomes. Nucleic Acids Res. 2000;28(1):27-30.

96. Kanehisa M. Toward understanding the origin and evolution of cellular organisms. Protein Sci. 2019;28(11):1947-51.

97. Kanehisa M, Furumichi M, Sato Y, Ishiguro-Watanabe M, Tanabe M. KEGG: integrating viruses and cellular organisms. Nucleic Acids Res. 2021;49(D1):D545-D51.
98. Betel D, Wilson M, Gabow A, Marks DS, Sander C. The microRNA.org resource: targets and expression. Nucleic Acids Res. 2008;36(Database issue):D149-53.

99. Allen E, Xie Z, Gustafson AM, Carrington JC. microRNA-directed phasing during trans-acting siRNA biogenesis in plants. Cell. 2005;121(2):207-21.

100. Shannon P, Markiel A, Ozier O, Baliga NS, Wang JT, Ramage D, et al. Cytoscape: a software environment for integrated models of biomolecular interaction networks. Genome Res. 2003;13(11):2498-504.

101. Yu G, Lam TT, Zhu H, Guan Y. Two methods for mapping and visualizing associated data on phylogeny using Ggtree. Mol Biol Evol. 2018;35(12):3041-3.

102. Hung JH, Weng Z. Sequence alignment and homology search with BLAST and ClustalW. Cold Spring Harb Protoc. 2016;2016(11). https:// doi.org/10.1101/pdb.prot093088.

103. Schefe $\mathrm{HH}$, Lehmann KE, Buschmann IR, Unger T, Funke-Kaiser H. Quantitative real-time RT-PCR data analysis: current concepts and the novel "gene expression's CT difference" formula. J Mol Med (Berl). 2006;84(11):901-10.

104. Jacob TR, Peres NT, Persinoti GF, Silva LG, Mazucato M, Rossi A, et al. rpb2 is a reliable reference gene for quantitative gene expression analysis in the dermatophyte Trichophyton rubrum. Med Mycol. 2012;50(4):368-77.

\section{Publisher's Note}

Springer Nature remains neutral with regard to jurisdictional claims in published maps and institutional affiliations.

Ready to submit your research? Choose BMC and benefit from

- fast, convenient online submission

- thorough peer review by experienced researchers in your field

- rapid publication on acceptance

- support for research data, including large and complex data types

- gold Open Access which fosters wider collaboration and increased citations

- maximum visibility for your research: over 100M website views per year

At BMC, research is always in progress.

Learn more biomedcentral.com/submissions 